\title{
Generalized Fuzzy Soft Connected Sets in Generalized Fuzzy Soft Topological Spaces
}

\author{
F.H. Khedr ${ }^{1}$, M. AZAB. Abd-Allah², and M.S Malfi ${ }^{3}$ \\ 1,2,3 Mathematics Department, Faculty of Science, Assiut University, Assiut 71516, Egypt \\ ${ }^{1}$ khedrfathi@gmail.com, ${ }^{2}$ mazab57@yahoo.com, ${ }^{3}$ mulfy_s76@yahoo.com
}

\section{ABSTRACT}

In this paper we introduce some types of generalized fuzzy soft separated sets and study some of their properties. Next, the notion of connectedness in fuzzy soft topological spaces due to Karata et al, Mahanta et al, and Kandil et al., extended to generalized fuzzy soft topological spaces. The relationship between these types of connectedness in generalized fuzzy soft topological spaces is investigated with the help of number of counter examples.

Keywords: Generalized fuzzy soft sets; generalized fuzzy soft topological space; generalized fuzzy soft separated sets; generalized fuzzy soft Q-separated sets; generalized fuzzy soft weakly separated sets; generalized fuzzy soft strongly separated sets; generalized fuzzy soft connected sets.

Date of Publication: 2018-06-30

DOI: $10.24297 / j a m . v 14 i 2.7461$

ISSN: $2347-1921$

Volume:14 Issue: 02

Journal: Journal of Advances in Mathematics

Website: https://cirworld.com

This work is licensed under a Creative Commons Attribution 4.0 International License. 


\section{INTRODUCTION}

The concept of soft sets was first introduced by Molodtsov [16] as a general mathematical tool for dealing with uncertain objects. Cagman et al. [2], Shabir et al. [20] introduced soft topological space independently. Maji et al. [13] introduced the concept of fuzzy soft set and some of its properties. Tanay and Kandemir [21] introduced the definition of a fuzzy soft topology over a subset of the initial universe set. Later, Roy and Samanta [18] gave the definition of fuzzy soft topology over the initial universe set. Karal and Ahmed [8] defined the notion of a mapping on classes of fuzzy soft sets.

Majumdar and Samanta [14] introduced the notion of generalized fuzzy soft set as a generalization of fuzzy soft sets and studied some of its basic properties. Chakraborty and Mukherjee. [3] gave the topological structure of generalized fuzzy soft sets. Khedr et al. [9] introduced the concept of a generalized fuzzy soft point, a generalized fuzzy soft base (subbase), a generalized fuzzy soft subspace. Khedr et al. [10] introduced the concept of a generalized fuzzy soft mapping on families of generalized fuzzy soft sets.

The notion of connectedness in fuzzy topological spaces has been studied by Ming and Ming [15], Zheng Chong You [23], Fatteh and Bassan [5], Saha [19], and Ajmal and Kohli [1]. In fuzzy soft setting, connectedness has been introduced by Mahanta et al. [12], Karata et al. [7] and Kandil et al. [6]

Khedr et al. [11] introduced the generalized fuzzy soft connectedness and generalized fuzzy soft $C_{i}$ connectedness $(i=1,2,3,4)$ in generalized fuzzy soft topological space and studied some of its basic properties.

In this paper, we extend the notion of connectedness of fuzzy soft topological spaces to generalized fuzzy soft topological spaces. In Section 3, we introduce different notions of generalized fuzzy soft separated sets and study the relationship between them. Section 4 is devoted to introduce the different notions of connectedness in generalized fuzzy soft topological spaces and study the implications that exist between them. Also, we study some characterizations of connectedness in generalized fuzzy soft setting.

\section{Preliminaries}

In this section, we will give some basic definitions and theorems about generalized fuzzy soft sets, generalized fuzzy soft topology and generalized fuzzy soft continuous mappings which will be needed in the sequel.

Definition 2.1. [22] Let $X$ be a non-empty set. A fuzzy set $A$ in $X$ is defined by a membership function $\mu_{A}: X \rightarrow$ $[0,1]$ whose value $\mu_{A}(x)$ represents the "grade of membership" of $x$ in $A$ for $x \in X$. The set of all fuzzy sets in a set $X$ is denoted by $I^{X}$, where $I$ is the closed unit interval $[0,1]$.

Definition 2.2. [22] If $A, B \in I^{X}$, then, we have:

(i) $A \leq B \Leftrightarrow \mu_{A}(x) \leq \mu_{B}(x), \forall x \in X$;

(ii) $A=B \Leftrightarrow \mu_{A}(x)=\mu_{B}(x), \forall x \in X$;

(iii) $C=A \vee B \Leftrightarrow \mu_{C}(x)=\max \left(\mu_{A}(x), \mu_{B}(x)\right), \forall x \in X$;

(iv) $D=A \wedge B \Leftrightarrow \mu_{D}(x)=\min \left(\mu_{A}(x), \mu_{B}(x)\right), \forall x \in X$;

(v) $E=A^{C} \Leftrightarrow \mu_{E}(x)=1-\mu_{A}(x), \forall x \in X$. 
Definition 2.3. [16] Let $X$ be an initial universe set and $E$ be a set of parameters. Let $P(X)$ denotes the power set of $X$ and $A \subseteq E$. A pair $(f, A)$ is called a soft set over $X$ if $f$ is a mapping from $A$ into $P(X)$, i.e., $f: A \rightarrow$ $P(X)$. In other words, a soft set is a parameterized family of subsets of the set $X$. For $e \in A, f(e)$ may be considered as the set of $e$-approximate elements of the soft set $(f, A)$.

Definition 2.4. [18] Let $X$ be an initial universe set and $E$ be a set of parameters. Let $A \subseteq E$. A fuzzy soft set $f_{A}$ over $X$ is a mapping from $E$ to $I^{X}$, i.e., $f_{A}: E \rightarrow I^{X}$, where $f_{A}(e) \neq \overline{0}$ if $e \in A \subseteq E$, and $f_{A}(e)=\overline{0}$ if $e \notin A_{i}$ where $\overline{0}$ denotes the empty fuzzy set in $X$.

Definition 2.5. [14] Let $X$ be a universal set of elements and $E$ be a universal set of parameters for $X$. Let $F$ : $E \rightarrow I^{X}$ and $\mu$ be a fuzzy subset of $E$, i.e., $\mu: E \rightarrow I$. Let $F_{\mu}$ be the mapping $F_{\mu}: E \rightarrow I^{X} \times I$ defined as follows: $F_{\mu}(e)=(F(e), \mu(e))$, where $F(e) \in I^{X}$ and $\mu(e) \in I$. Then $F_{\mu}$ is called a generalised fuzzy soft set (GFSS in short) over $(X, E)$. The family of all generalized fuzzy soft sets over $(X, E)$ is denoted by $\operatorname{GFSS}(X, E)$.

Definition 2.6. [14] Let $F_{\mu}$ and $G_{\delta}$ be two GFSSs over $(X, E) . F_{\mu}$ is said to be a GFS subset of $G_{\delta}$ or $G_{\delta}$ is said to be a GFS super set of $F_{\mu}$ denoted by $F_{\mu} \subseteq G_{\delta}$ if

(i) $\mu$ is a fuzzy subset of $\delta$;

(ii) $F(e)$ is also a fuzzy subset of $G(e), \forall e \in E$.

Definition 2.7. [14] Let $F_{\mu}$ be a GFSS over $(X, E)$. The generalized fuzzy soft complement of $F_{\mu}$, denoted by $F_{\mu}^{c}$, is defined by $F_{\mu}^{c}=G_{\delta}$, where $\delta(e)=\mu^{c}(e)$ and $G(e)=F^{c}(e), \forall e \in E$.

Obviously $\left(F_{\mu}^{c}\right)^{\mathrm{c}}=F_{\mu}$.

Definition 2.8. [3] Let $F_{\mu}$ and $G_{\delta}$ be two GFSSs over $(X, E)$. The generalized fuzzy soft union (GFS union, in short) of $F_{\mu}$ and $G_{\delta}$, denoted by $F_{\mu} \sqcup G_{\delta}$, is The GFSS $H_{v}$, defined as $H_{v}: E \rightarrow I^{X} \times I$ such that

$H_{v}(e)=(H(e), v(e))$, where $H(e)=F(e) \vee G(e)$ and $v(e)=\mu(e) \vee \delta(e), \forall e \in E$.

Let $\left\{\left(F_{\mu}\right)_{\lambda}, \lambda \in \nabla\right\}$, where $\nabla$ is an index set, be a family of GFSSs. The GFS union of these family, denoted by $\sqcup_{\lambda \in \Lambda}\left(F_{\mu}\right)_{\lambda}$, is The GFSS $H_{v}$, defined as $H_{v}: E \rightarrow I^{X} \times I$ such that $H_{v}(e)=(H(e), v(e))$, where $H(e)=$ $\mathrm{V}_{\lambda \in \nabla}(F(e))_{\lambda}$, and $v(e)=\mathrm{V}_{\lambda \in \nabla}(\mu(e))_{\lambda}, \forall e \in E$.

Definition 2.9. [3] Let $F_{\mu}$ and $G_{\delta}$ be two GFSSs over $(X, E)$. The generalized fuzzy soft Intersection (GFS Intersection, in short) of $F_{\mu}$ and $G_{\delta}$, denoted by $F_{\mu} \sqcap G_{\delta}$, is the GFSS $M_{\sigma}$, defined as $M_{\sigma}: E \rightarrow I^{X} \times I$ such that

$M_{\sigma}(e)=(M(e), \sigma(e))$, where $M(e)=F(e) \wedge G(e)$ and $\sigma(e)=\mu(e) \wedge \delta(e), \forall e \in E$.

Let $\left\{\left(F_{\mu}\right)_{\lambda}, \lambda \in \nabla\right\}$, where $\nabla$ is an index set, be a family of GFSSs. The GFS Intersection of these family, denoted by $\Pi_{\lambda \in \nabla}\left(F_{\mu}\right)_{\lambda}$, is the GFSS $M_{\sigma}$, defined as $M_{\sigma}: E \rightarrow I^{X} \times I$ such that $M_{\sigma}(e)=(M(e), \sigma(e))$, where $M(e)=\Lambda_{\lambda \in \nabla}(F(e))_{\lambda}$, and $\sigma(e)=\Lambda_{\lambda \in \nabla}(\mu(e))_{\lambda}, \forall e \in E$.

Theorem 2.1. [3] Let $\left\{\left(F_{\mu}\right)_{\lambda}, \lambda \in \nabla\right\} \subseteq \operatorname{GFSS}(X, E)$. Then the following statements hold,

$\left[\sqcup_{\lambda \in \nabla}\left(F_{\mu}\right)_{\lambda}, \lambda \in \nabla\right]^{c}=\Pi_{\lambda \in \nabla}\left(F_{\mu}\right)_{\lambda}^{c}$

$\left[\Pi_{\lambda \in \nabla}\left(F_{\mu}\right)_{\lambda}, \lambda \in \nabla\right]^{c}=\sqcup_{\lambda \in \nabla}\left(F_{\mu}\right)_{\lambda}^{c}$ 
Definition 2.10. [14] A GFSS is said to be a generalized null fuzzy soft set, denoted by $\tilde{0}_{\theta}$, if $\tilde{0}_{\theta}: E \rightarrow I^{X} \times I$ such that $\tilde{0}_{\theta}(e)=(\tilde{0}(e), \theta(e))$ where $\tilde{0}(e)=\overline{0} \forall e \in E$ and $\theta(e)=0 \quad \forall e \in E$ ( Where $\overline{0}(x)=0, \forall x \in X$ ).

Definition 2.11. [14] A GFSS is said to be a generalized absolute fuzzy soft set, denoted by $\tilde{1}_{\Delta}$, if $\tilde{1}_{\Delta}: E \rightarrow$ $I^{X} \times I$, where $\tilde{1}_{\Delta}(e)=(\tilde{1}(e), \Delta(e))$ is defined by $\tilde{1}(e)=\overline{1}, \forall e \in E$ and $\Delta(e)=1, \forall e \in E$ ( Where $\overline{1}(x)=1, \forall x \in$ $X)$.

Definition 2.12. [3] Let $T$ be a collection of generalized fuzzy soft sets over $(X, E)$. Then $T$ is said to be a generalized fuzzy soft topology ( $G F S$ topology in short) over $(X, E)$ if the following conditions are satisfied:

(i) $\tilde{0}_{\theta}$ and $\tilde{1}_{\Delta}$ are in $T$;

(ii) Arbitrary GFS unions of members of $T$ belong to $T$;

(iii) Finite GFS intersections of members of $T$ belong to $T$.

The triple $(X, T, E)$ is called a generalized fuzzy soft topological space (GFST-space in short) over $(X, E)$.

The members of $T$ are called generalized fuzzy soft open sets [ GFS open in short] in $(X, T, E)$.

Definition 2.13 [3] Let $(X, T, E)$ be a GFST -space. A GFSS $F_{\mu}$ over $(X, E)$ is said to be a generalized fuzzy soft closed set in $X$ [GFS closed in short], if its complement $F_{\mu}^{c}$ is GFS open. The collection of all GFS closed sets will be denoted by $T^{c}$.

Definition 2.14. [3] Let $(X, T, E)$ be a GFST -space and $F_{\mu} \in \operatorname{GFSS}(X, E)$. The generalized fuzzy soft closure of $F_{\mu}$, denoted by $\operatorname{cl}\left(F_{\mu}\right)$, is the intersection of all GFS closed supper sets of $F_{\mu}$. i.e., $\operatorname{cl}\left(F_{\mu}\right)=\Pi\left\{H_{v}: H_{v} \in T^{c}, F_{\mu}\right.$ 드 $\left.H_{v}\right\}$. Clearly, $\operatorname{cl}\left(F_{\mu}\right)$ is the smallest $G F S$ closed set over $(X, E)$ which contains $F_{\mu}$.

Definition 2.15. [9] The generalized fuzzy soft set $F_{\mu} \in G F S(X, E)$ is called a generalized fuzzy soft point (GFS point in short) if there exist $e \in E$ and $x \in X$ such that

(i) $F(e)(x)=\alpha(0<\alpha \leq 1)$ and $F(e)(y)=0$ for all $y \in X-\{x\}$,

(ii) $\mu(e)=\lambda(0<\lambda \leq 1)$ and $\mu\left(e^{\prime}\right)=0$ for all $e^{\prime} \in E-\{e\}$. We denote this generalized fuzzy soft point $F_{\mu}=$ $\left(x_{\alpha}, e_{\lambda}\right)$.

$(x, e)$ and $(\alpha, \lambda)$ are called respectively, the support and the value of $\left(x_{\alpha}, e_{\lambda}\right)$.

Definition 2.16. [9] Let $F_{\mu}$ be a GFSS over $(X, E)$. We say that $\left(x_{\alpha}, e_{\lambda}\right) \widetilde{\in} F_{\mu}$ read as $\left(x_{\alpha}, e_{\lambda}\right)$ belongs to the GFSS $F_{\mu}$ if for the element $e \in E, \alpha \leq F(e)(x)$ and $\lambda \leq \mu(e)$.

Definition 2.17. [17] For any two GFSSs $F_{\mu}$ and $G_{\delta}$ over $(X, E) . F_{\mu}$ is said to be a generalized fuzzy soft quasicoincident with $G_{\delta}$, denoted by $F_{\mu} q G_{\delta}$, if there exist $e \in E$ and $x \in X$ such that $F(e)(x)+G(e)(x)>1$ and $\mu(e)+\delta(e)>1$.

If $F_{\mu}$ is not generalized fuzzy soft quasi-coincident with $G_{\delta}$, then we write $F_{\mu} \bar{q} G_{\delta}$, i.e., for every $e \in E$ and $x \in X$, $F(e)(x)+G(e)(x) \leq 1$ or for every $e \in E$ and $x \in X, \mu(e)+\delta(e) \leq 1$.

Definition 2.18. [17] Let $\left(x_{\alpha}, e_{\lambda}\right)$ be a GFS point and $F_{\mu}$ be a GFSS over $(X, E) .\left(x_{\alpha}, e_{\lambda}\right)$ is said to be generalized fuzzy soft quasi-coincident with $F_{\mu}$ denoted by $\left(x_{\alpha}, e_{\lambda}\right) q F_{\mu}$, if and only if there exists an element $e \in E$ such that $\alpha+F(e)(x)>1$ and $\lambda+\mu(e)>1$.

Theorem 2.2. [17] Let $F_{\mu}$ and $G_{\delta}$ are GFSSs over $(X, E)$. Then the following are hold: 
$(1) F_{\mu} \sqsubseteq G_{\delta} \Leftrightarrow F_{\mu} \bar{q}\left(G_{\delta}\right)^{c} ;$

(2) $F_{\mu} q G_{\delta} \Rightarrow F_{\mu} \sqcap G_{\delta} \neq \tilde{0}_{\theta}$;

(3) $\left(x_{\alpha}, e_{\lambda}\right) \bar{q} F_{\mu} \Leftrightarrow\left(x_{\alpha}, e_{\lambda}\right) \widetilde{\in}\left(F_{\mu}\right)^{c}$;

(4) $F_{\mu} \bar{q}\left(F_{\mu}\right)^{c}$.

Definition 2.19. [10] Let $\operatorname{GFSS}(X, E)$ and $\operatorname{GFSS}(Y, K)$ be the families of all generalized fuzzy soft sets over $(X, E)$ and $(Y, K)$, respectively. Let $u: X \rightarrow Y$ and $p: E \rightarrow K$ be two functions. Then a mapping $f_{u p}$ : $\operatorname{GFSS}(X, E) \rightarrow \operatorname{GFSS}(Y, K)$ is defined as follows: for a generalized fuzzy soft set $F_{\mu} \in \operatorname{GFSS}(X, E), \forall k \in$ $p(E) \subseteq K$ and $y \in Y$,

$$
f_{u p}\left(F_{\mu}\right)(k)(y)= \begin{cases}\left(\mathrm{V}_{x \in u^{-1}(y)} \mathrm{V}_{e \in p^{-1}(k)} F(e)(x), \mathrm{V}_{e \in p^{-1}(k)} \mu(e)\right) & \text { if } u^{-1}(y) \neq \varphi, p^{-1}(k) \neq \varphi, \\ (0,0), & \text { otherwise. }\end{cases}
$$

$f_{u p}$ is called a generalized fuzzy soft mapping [GFS mapping in short] and $f_{u p}\left(F_{\mu}\right)$ is called a GFS image of a GFSS $F_{\mu}$.

Definition 2.20. [10] Let $u: X \rightarrow Y$ and $p: E \rightarrow K$ be mappings. Let $f_{u p}: \operatorname{GFSS}(X, E) \rightarrow \operatorname{GFSS}(Y, K)$ be a GFS mapping and $G_{\delta} \in \operatorname{GFSS}(Y, K)$. Then, $f_{u p}^{-1}\left(G_{\delta}\right) \in \operatorname{GFSS}(X, E)$, defined as follows:

$$
f_{u p}^{-1}\left(G_{\delta}\right)(e)(x)=(G(p(e))(u(x)), \delta(p(e))), \text { for } e \in E, x \in X .
$$

$f_{u p}^{-1}\left(G_{\delta}\right)$ is called a GFS inverse image of $G_{\delta}$.

If $u$ and $p$ are injective then the generalized fuzzy soft mapping $f_{u p}$ is said to be injective. If $u$ and $p$ are surjective then the generalized fuzzy soft mapping $f_{u p}$ is said to be surjective. The generalized fuzzy soft mapping $f_{u p}$ is called constant, if $u$ and $p$ are constant.

Definition 2.21. [10] Let $\left(X, T_{1}, E\right)$ and $\left(Y, T_{2}, K\right)$ be two $G F S T$-spaces, and $f_{u p}:\left(X, T_{1}, E\right) \rightarrow\left(Y, T_{2}, K\right)$ be a GFS mapping. Then $f_{\text {up }}$ is called

(1) generalized fuzzy soft continuous [GFS-continuous in short] if $f_{u p}^{-1}\left(G_{\delta}\right) \in T_{1}$ for all $G_{\delta} \in T_{2}$.

(2) generalized fuzzy soft open [ GFS open in short] if $f_{u p}\left(F_{\mu}\right) \in T_{2}$ for each $F_{\mu} \in T_{1}$.

Definition 2.22. [11] Let $(X, T, E)$ be a $G F S T$-space and $F_{\mu} \in G F S(X, E)$. Then, $F_{\mu}$ is called

i. GFSC $C_{1}$-connected if and only if it does not exist two non null GFS open sets $H_{v}$ and $K_{\gamma}$ such that $F_{\mu} \sqsubseteq H_{\nu} \sqcup$ $K_{\gamma}, H_{v} \sqcap K_{\gamma} \sqsubseteq F_{\mu}^{c}, F_{\mu} \sqcap H_{v} \neq \tilde{0}_{\theta}$ and $F_{\mu} \sqcap K_{\gamma} \neq \tilde{0}_{\theta}$.

ii. $G F S C_{2}$-connected if and only if it does not exist two non null GFS open sets $H_{v}$ and $K_{\gamma}$ such that $F_{\mu} \sqsubseteq H_{\nu} \sqcup$ $K_{\gamma}, \quad F_{\mu} \sqcap H_{v} \sqcap K_{\gamma}=\tilde{0}_{\theta}, F_{\mu} \sqcap H_{v} \neq \tilde{0}_{\theta}$ and $F_{\mu} \sqcap K_{\gamma} \neq \tilde{0}_{\theta}$.

iii. $G F S C_{3}$-connected if and only if it does not exist two non null GFS open sets $H_{v}$ and $K_{\gamma}$ such that $F_{\mu} \sqsubseteq H_{\nu} \sqcup$ $K_{\gamma}, H_{v} \sqcap K_{\gamma} \subseteq F_{\mu}^{c}, H_{v} \nsubseteq F_{\mu}^{c}$ and $K_{\gamma} \nsubseteq F_{\mu}^{c}$.

iv. GFSC $C_{4}$-connected if and only if it does not exist two non null GFS open sets $H_{v}$ and $K_{\gamma}$ such that $F_{\mu} \sqsubseteq H_{v} \sqcup$ $K_{\gamma}, F_{\mu} \sqcap H_{v} \sqcap K_{\gamma}=\tilde{0}_{\theta}, H_{v} \nsubseteq F_{\mu}^{c}$ and $K_{\gamma} \nsubseteq F_{\mu}^{c}$.

Otherwise, $F_{\mu}$ is called not $G F S C_{i}$-connected set for $i=1,2,3,4$. 
In the above definition, if we take $\tilde{1}_{\Delta}$ instead of $F_{\mu}$, then the $\operatorname{GFST}$-space $(X, T, E)$ is called $G F S C_{i}$-connected space $(i=1,2,3,4)$.

Remark 2.1. [11] The relationship between $G F S C_{i}$-connectedness $(i=1,2,3,4)$ can be described by the following diagram:

$$
\begin{aligned}
& G_{F S C} \Rightarrow G_{1} S_{2} \\
& \Downarrow \quad \Downarrow \\
& \mathrm{GFSC}_{3} \Rightarrow \mathrm{GFSC}_{4}
\end{aligned}
$$

Remark 2.2. [11] The reverse implications is not true in general (see Examples 4.2, 4.3, 4.4, 4.5, 4.6 in [11]).

\section{GENERALIZED FUZZY SOFT SEPARATED SETS IN GENERALIZED FUZZY SOFT TOPOLOGICAL SPACES}

In this section, we will introduce different notions of generalized fuzzy soft separated sets and study the relation between these notions. Also, we will investigate the characterizations of the generalized fuzzy soft separated sets.

Definition 3.1. Two non-null GFSS sets $F_{\mu}$ and $G_{\delta}$ in $G F S T$-space $(X, T, E)$ are said to be generalized fuzzy soft $Q$-separated [GFS $Q$-separated, in short] if $\operatorname{cl}\left(F_{\mu}\right) \sqcap G_{\delta}=F_{\mu} \sqcap \operatorname{cl}\left(G_{\delta}\right)=\tilde{0}_{\theta}$.

Theorem 3.1. Let $(X, T, E)$ be a GFST-space, $F_{\mu}$ and $G_{\delta}$ be two GFS closed sets in $(X, E)$. Then $F_{\mu}$ and $G_{\delta}$ are GFS $Q$-separated sets if and only if $F_{\mu} \sqcap G_{\delta}=\tilde{0}_{\theta}$.

Proof. Suppose that $F_{\mu}$ and $G_{\delta}$ are $G F S Q$-separated sets. Then $\operatorname{cl}\left(F_{\mu}\right) \sqcap G_{\delta}=F_{\mu} \sqcap \operatorname{cl}\left(G_{\delta}\right)=\tilde{0}_{\theta}$. Since $F_{\mu}$ and $G_{\delta}$ are $G F S$ closed sets then, $F_{\mu} \sqcap G_{\delta}=\tilde{0}_{\theta}$.

Conversely, let $F_{\mu} \sqcap G_{\delta}=\tilde{0}_{\theta}$. Since $F_{\mu}$ and $G_{\delta}$ are GFS closed sets, then $\operatorname{cl}\left(F_{\mu}\right) \sqcap G_{\delta}=F_{\mu} \sqcap G_{\delta}=\tilde{0}_{\theta}$ and $F_{\mu} \sqcap$ $\operatorname{cl}\left(G_{\delta}\right)=F_{\mu} \sqcap G_{\delta}=\tilde{0}_{\theta}$. It follows that, $F_{\mu}$ and $G_{\delta}$ are GFS $Q$-separated sets.

Theorem 3.2. Let $H_{v}, K_{\gamma}$ be GFS $Q$-separated sets of $G F S T$-space $(X, T, E)$ and $F_{\mu} \sqsubseteq H_{v}, G_{\delta} \subseteq K_{\gamma}$. Then, $F_{\mu}, G_{\delta}$ are GFSQ -separated sets.

Proof. Let $F_{\mu} \sqsubseteq H_{v}$. Then, $\operatorname{cl}\left(F_{\mu}\right) \subseteq \operatorname{cl}\left(H_{v}\right)$. It follows that, $\operatorname{cl}\left(F_{\mu}\right) \sqcap G_{\delta} \sqsubseteq \operatorname{cl}\left(F_{\mu}\right) \sqcap K_{\gamma} \sqsubseteq c l\left(H_{v}\right) \sqcap K_{\gamma}=\tilde{0}_{\theta}$. Also, since $G_{\delta} \sqsubseteq K_{\gamma}$. Then, $\operatorname{cl}\left(G_{\delta}\right) \subseteq \operatorname{cl}\left(K_{\gamma}\right)$. Hence, $F_{\mu} \sqcap \operatorname{cl}\left(G_{\delta}\right) \subseteq H_{v} \sqcap \operatorname{cl}\left(K_{\gamma}\right)=\tilde{0}_{\theta}$. Thus $F_{\mu}, G_{\delta}$ are GFSQ -separated sets.

Definition 3.2. Two non- null GFSSs $F_{\mu}$ and $G_{\delta}$ in $G F S T$-space $(X, T, E)$ are said to be generalized fuzzy soft weakly separated [ in short, GFS weakly separated] if $c l\left(F_{\mu}\right) \bar{q} G_{\delta}$ and $F_{\mu} \bar{q} c l\left(G_{\delta}\right)$.

Theorem 3.3. Let $(X, T, E)$ be a GFST-space and $F_{\mu}, G_{\delta} \in G F S(X, E)$. Then, $F_{\mu}$ and $G_{\delta}$ are $G F S$ weakly separated sets if and only if there exist GFS open sets $H_{v}$ and $K_{\gamma}$ such that $F_{\mu} \subseteq H_{\nu}, G_{\delta} \subseteq K_{\gamma}$, and $F_{\mu} \bar{q} K_{\gamma}$ and $G_{\delta} \bar{q} H_{v}$.

Proof. Let $F_{\mu}$ and $G_{\delta}$ are GFS weakly separated sets in $(X, T, E)$. Then $\operatorname{cl}\left(F_{\mu}\right) \bar{q} G_{\delta}$ and $F_{\mu} \bar{q} c l\left(G_{\delta}\right)$. Therefore, $G_{\delta} \subseteq$ $\left[\operatorname{cl}\left(F_{\mu}\right)\right]^{c}$ and $F_{\mu} \subseteq\left[\operatorname{cl}\left(G_{\delta}\right)\right]^{c}$. Taking $H_{v}=\left[\operatorname{cl}\left(G_{\delta}\right)\right]^{c}$ and $K_{\gamma}=\left[\operatorname{cl}\left(F_{\mu}\right)\right]^{c}$. Then, $H_{v}, K_{\gamma} \in T, F_{\mu} \bar{q} K_{\gamma}$ and $G_{\delta} \bar{q} H_{v}$. The converse is obvious.

Remark 3.1. From Definitions 3.1, 3.2 if $F_{\mu}$ and $G_{\delta}$ are GFS $Q$-separated sets, then $F_{\mu}$ and $G_{\delta}$ are GFS weakly separated sets. 
Remark 3.2. Two GFS weakly separated sets may not be GFS $Q$-separated as shown by the following example.

Example 3.1. Let $X=\left\{x_{1}, x_{2}\right\}, E=\left\{e_{1}, e_{2}\right\}$ and $T=\left\{\tilde{0}_{\theta}, \tilde{1}_{\Delta},\left\{\left(e_{1}=\left\{\frac{x_{1}}{0.3}, \frac{x_{2}}{0.2}\right\}, 0.4\right),\left(e_{2}=\left\{\frac{x_{1}}{0.5}, \frac{x_{2}}{0.3}\right\}, 0.6\right)\right\}\right\}$ be a GFS topology over $(X, E)$. If $F_{\mu}=\left\{\left(e_{1}=\left\{\frac{x_{1}}{0.1}\right\}, 0.2\right)\right\}$ and $G_{\delta}=\left\{\left(e_{2}=\left\{\frac{x_{1}}{0.1}, \frac{x_{2}}{0.1}\right\}, 0.3\right)\right\}$. Then $F_{\mu}$ and $G_{\delta}$ are $G F S$ weakly separated sets, but $F_{\mu}$ and $G_{\delta}$ are not GFS $Q$-separated.

Definition 3.3. Two non- null GFSSS $F_{\mu}$ and $G_{\delta}$ in $G F S T$-space $(X, T, E)$ are said to be generalized fuzzy soft separated [ in short, GFS separated] if there exist GFS open sets $H_{v}$ and $K_{\gamma}$ such that $F_{\mu} \sqsubseteq H_{v}, G_{\delta} \subseteq K_{\gamma}$ and $F_{\mu} \sqcap$ $K_{\gamma}=G_{\delta} \sqcap H_{v}=\tilde{0}_{\theta}$.

Remark 3.3. Two GFS separated sets are GFS weakly separated sets.

Proof. From Definitions 3.3 and Theorem 3.3 it follows that.

Remark 3.4. Two GFS weakly separated sets may not be GFS separated. In fact, $F_{\mu}$ and $G_{\delta}$ defined in Example 3.1, are GFS weakly separated, but not GFS separated.

Remark 3.5. The notions of GFS separated sets and GFS Q -separated are independent to each others as shown by the following example.

Example 3.2. Let $X=\left\{x_{1}, x_{2}\right\}, E=\left\{e_{1}, e_{2}\right\}$ and

$T=\left\{\tilde{0}_{\theta}, \tilde{1}_{\Delta}, H_{v}=\left\{\left(e_{1}=\left\{\frac{x_{1}}{0.5}\right\}, 0.3\right)\right\}, K_{\gamma}=\left\{\left(e_{2}=\left\{\frac{x_{2}}{0.5}\right\}, 0.3\right)\right\}, H_{v} \sqcup K_{\gamma}\right\}$ be a GFS topology over $(X, E)$.

If $F_{\mu}=\left\{\left(e_{1}=\left\{\frac{x_{1}}{0.2}\right\}, 0.1\right)\right\}$ and $G_{\delta}=\left\{\left(e_{2}=\left\{\frac{x_{2}}{0.2}\right\}, 0.1\right)\right\}$. Then there exist GFS open sets $H_{v}$ and $K_{\gamma}$ such that $F_{\mu} \subseteq$ $H_{v}, G_{\delta} \subseteq K_{\gamma}$ and $F_{\mu} \sqcap K_{\gamma}=G_{\delta} \sqcap H_{v}=\tilde{0}_{\theta}$. So, $F_{\mu}$ and $G_{\delta}$ are $G F S$ separated sets.

But $F_{\mu}$ and $G_{\delta}$ are not GFS $Q$-separated. Since, $\operatorname{cl}\left(F_{\mu}\right)=\left\{\left(e_{1}=\left\{\frac{x_{1}}{0.5}, \frac{x_{2}}{1}\right\}, 0.7\right),\left(e_{2}=\left\{\frac{x_{1}}{1}, \frac{x_{2}}{0.5}\right\}, 0.7\right)\right\}$ and $\operatorname{cl}\left(F_{\mu}\right) \sqcap$ $G_{\delta} \neq \tilde{0}_{\theta}$.

Example 3.3. Let $X=\left\{x_{1}, x_{2}\right\}, E=\left\{e_{1}, e_{2}\right\}$ and

$T=\left\{\tilde{0}_{\theta}, \tilde{1}_{\Delta},\left\{\left(e_{1}=\left\{\frac{x_{1}}{0.3}, \frac{x_{2}}{0.2}\right\}, 0.4\right),\left(e_{2}=\left\{\frac{x_{1}}{1}, \frac{x_{2}}{1}\right\}, 1\right)\right\},\left\{\left(e_{1}=\left\{\frac{x_{1}}{1}, \frac{x_{2}}{1}\right\}, 1\right),\left(e_{2}=\left\{\frac{x_{1}}{0.1}, \frac{x_{2}}{0.4}\right\}, 0.3\right)\right\}\right.$,

$\left.\left\{\left(e_{1}=\left\{\frac{x_{1}}{0.3}, \frac{x_{2}}{0.2}\right\}, 0.4\right),\left(e_{2}=\left\{\frac{x_{1}}{0.1}, \frac{x_{2}}{0.4}\right\}, 0.3\right)\right\}\right\}$ be a GFS topology over $(X, E)$. Let $F_{\mu}=\left\{\left(e_{1}=\left\{\frac{x_{1}}{0.2}\right\}, 0.3\right)\right\}$ and $G_{\delta}=\left\{\left(e_{2}=\left\{\frac{x_{2}}{0.3}\right\}, 0.2\right)\right\}$. Then $F_{\mu}$ and $G_{\delta}$ are GFS $Q$-separated sets, but not GFS separated.

Definition 3.4. Let $F_{\mu} \in G F S(X, E)$. The generalized fuzzy soft support ( in short, GFS support) of $F_{\mu}$ defined by $S\left(F_{\mu}\right)$ is the set, $S\left(F_{\mu}\right)=\{x \in X, e \in E: F(e)(x)>0$ and $\mu(e)>0\}$.

Definition 3.5. Two non- null GFSSS $F_{\mu}$ and $G_{\delta}$ are said to be $G F S$ quasi-coincident with respect to $F_{\mu}$ if $F(e)(x)+G(e)(x)>1$ and $\mu(e)+\delta(e)>1$ for every $x, e \in S\left(F_{\mu}\right)$.

Definition 3.6. Two non- null GFSSS $F_{\mu}$ and $G_{\delta}$ in a $\operatorname{GFST}$-space $(X, T, E)$ are said to be generalized fuzzy soft strongly separated [ in short, GFS strongly separated] if there exist GFS open sets $H_{v}$ and $K_{\gamma}$ such that

i. $F_{\mu} \sqsubseteq H_{v}, G_{\delta} \sqsubseteq K_{\gamma}$ and $F_{\mu} \sqcap K_{\gamma}=G_{\delta} \sqcap H_{v}=\tilde{0}_{\theta}$

ii. $F_{\mu}$ and $H_{v}$ are $G F S$ quasi-coincident with respect to $F_{\mu}$

iii. $G_{\delta}$ and $K_{\gamma}$ are $G F S$ quasi-coincident with respect to $G_{\delta}$. 
Remark 3.6. From Definitions 3.3 and Remark 3.3 if $F_{\mu}$ and $G_{\delta}$ are $G F S$ strongly separated, then $F_{\mu}$ and $G_{\delta}$ are GFS separated and GFS weakly separated.

Remark 3.7. Two GFS separated sets may not be GFS strongly separated as shown by the following example.

Example 3.4. Let $X=\left\{x_{1}, x_{2}\right\}, E=\left\{e_{1}, e_{2}\right\}$ and

$T=\left\{\tilde{0}_{\theta}, \tilde{1}_{\Delta},\left\{\left(e_{1}=\left\{\frac{x_{1}}{0.3}, \frac{x_{2}}{0.2}\right\}, 0.3\right)\right\},\left\{\left(e_{2}=\left\{\frac{x_{1}}{0.2}, \frac{x_{2}}{0.2}\right\}, 0.4\right)\right\},\left\{\left(e_{1}=\left\{\frac{x_{1}}{0.3}, \frac{x_{2}}{0.2}\right\}, 0.3\right),\left(e_{2}=\left\{\frac{x_{1}}{0.2}, \frac{x_{2}}{0.2}\right\}, 0.4\right)\right\}\right\}$ be a $G F S$ topology over $(X, E)$. If $F_{\mu}=\left\{\left(e_{1}=\left\{\frac{x_{1}}{0.1}\right\}, 0.2\right)\right\}$ and $G_{\delta}=\left\{\left(e_{2}=\left\{\frac{x_{2}}{0.2}\right\}, 0.3\right)\right\}$. Then $F_{\mu}$ and $G_{\delta}$ are GFS separated sets, but not GFS strongly separated.

Remark 3.8. The notions of GFS $Q$-separated and GFS strongly separated are independent to each others as shown by the following example:

Example 3.5. In Example 3.3, $F_{\mu}$ and $G_{\delta}$ are $G F S Q$-separated sets, but not GFS strongly separated.

Example 3.6. Let $X=\left\{x_{1}, x_{2}\right\}, E=\left\{e_{1}, e_{2}\right\}$ and

$T=\left\{\tilde{0}_{\theta}, \tilde{1}_{\Delta},\left\{\left(e_{1}=\left\{\frac{x_{1}}{0.7}, \frac{x_{2}}{0.2}\right\}, 0.8\right)\right\},\left\{\left(e_{2}=\left\{\frac{x_{1}}{0.2}, \frac{x_{2}}{0.7}\right\}, 0.6\right)\right\},\left\{\left(e_{1}=\left\{\frac{x_{1}}{0.7}, \frac{x_{2}}{0.2}\right\}, 0.8\right),\left(e_{2}=\left\{\frac{x_{1}}{0.2}, \frac{x_{2}}{0.7}\right\}, 0.6\right)\right\}\right\}$ be a GFS topology over $(X, E)$. Let $F_{\mu}=\left\{\left(e_{1}=\left\{\frac{x_{1}}{0.5}\right\}, 0.6\right)\right\}$ and $G_{\delta}=\left\{\left(e_{2}=\left\{\frac{x_{2}}{0.4}\right\}, 0.5\right)\right\}$. Then $F_{\mu}$ and $G_{\delta}$ are GFS strongly separated, but not GFS $Q$-separated.

Remark 3.9. In $G F S T$-space $(X, T, E)$ the relationship between different notions of generalized fuzzy soft separated sets can be discribed by the following diagram.

GFS strongly separated

$\Downarrow$

GFS separated

$\Downarrow$

GFS $Q$ - separated $\Rightarrow$ GFS weakly separated

Theorem 3.4. Let $F_{\mu}$ and $G_{\delta}$ are GFS $Q$-separated (respectively, separated, strongly separated, weakly separated) sets in $(X, E)$ and $H_{v} \subseteq F_{\mu}, K_{\gamma} \subseteq G_{\delta}$. Then, $H_{v}$ and $K_{\gamma}$ are GFS $Q$-separated (respectively, separated, strongly separated, weakly separated) sets in $(X, E)$.

Proof. As a sample, we will prove the case GFS $Q$-separated. Let $F_{\mu}$ and $G_{\delta}$ are GFS $Q$-separated in $(X, E)$. Then, $\operatorname{cl}\left(F_{\mu}\right) \sqcap G_{\delta}=F_{\mu} \sqcap \operatorname{cl}\left(G_{\delta}\right)=\tilde{0}_{\theta}$. Since $H_{v} \sqsubseteq F_{\mu}, K_{\gamma} \sqsubseteq G_{\delta}$, then

$\operatorname{cl}\left(H_{v}\right) \sqcap K_{\gamma}=H_{v} \sqcap \operatorname{cl}\left(K_{\gamma}\right)=\tilde{0}_{\theta}$, therefore, $H_{v}$ and $G_{\delta}$ are $G F S Q$-separated set in $(X, E)$.

Theorem 3.5. Let $(X, T, E)$ be a GFST - space and $F_{\mu}, G_{\delta} \in G F S(X, E)$. Then, $F_{\mu}$ and $G_{\delta}$ are $G F S Q$-separated in $(X, E)$ if and only if there exist GFS closed sets $H_{v}$ and $K_{\gamma}$ such that $F_{\mu} \sqsubseteq H_{v}, G_{\delta} \sqsubseteq K_{\gamma}$ and $F_{\mu} \sqcap K_{\gamma}=G_{\delta} \sqcap H_{v}=$ $\tilde{0}_{\theta}$.

Proof. Let $F_{\mu}$ and $G_{\delta}$ are GFS $Q$-separated in $(X, E)$. Then, $\operatorname{cl}\left(F_{\mu}\right) \sqcap G_{\delta}=F_{\mu} \sqcap \operatorname{cl}\left(G_{\delta}\right)=\tilde{0}_{\theta}$. Taking $H_{v}=\operatorname{cl}\left(F_{\mu}\right)$ and $K_{\gamma}=c l\left(G_{\delta}\right)$. Therefore, $H_{v}$ and $K_{\gamma}$ are GFS closed sets in $(X, E)$ such that $F_{\mu} \sqsubseteq H_{v}, G_{\delta} \subseteq K_{\gamma}$ and $F_{\mu} \sqcap K_{\gamma}=$ $G_{\delta} \sqcap H_{v}=\tilde{0}_{\theta}$. The converse is obvious. 
Definition 3.7. Let $(X, T, E)$ be a GFST -space over $(X, E)$ and $G_{\delta}$ be GFS subset of $(X, E)$. Then $T_{G_{\delta}}=\left\{G_{\delta} \Pi\right.$ $\left.F_{\mu}: F_{\mu} \in T\right\}$ is called a GFS relative topology and $\left(G_{\delta}, T_{G_{\delta}}, E\right)$ is called a GFS subspace of $(X, T, E)$. If $G_{\delta} \in T$ (resp, $\left.G_{\delta} \in T^{c}\right)$ then $\left(G_{\delta}, T_{G_{\delta}}, E\right)$ is called generalized fuzzy soft open (resp. closed) subspace of $(X, T, E)$.

Theorem 3.6. Let $(X, T, E)$ be a $G F S T$-space and $G_{\delta} \subseteq F_{\mu} \widetilde{\in} G F S S(X, E)$.Then, $\operatorname{cl}_{F_{\mu}}\left(G_{\delta}\right)=\operatorname{cl}\left(G_{\delta}\right) \sqcap F_{\mu}$. Where $c l_{F_{\mu}}\left(G_{\delta}\right)$ denotes the GFS closure in the GFS subspace $\left(F_{\mu}, T_{F_{\mu}}, E\right)$.

Proof. We know $\operatorname{cl}\left(G_{\delta}\right)$ is $G F S$ closed set in $(X, T, E) \Rightarrow \operatorname{cl}\left(G_{\delta}\right) \sqcap F_{\mu}$ is $G F S$ closed set in $\left(F_{\mu}, T_{F_{\mu}}, E\right)$.

Now, $G_{\delta} \subseteq \operatorname{cl}\left(G_{\delta}\right) \sqcap F_{\mu}$ and $G F S$ closure of $G_{\delta}$ in $\left(F_{\mu}, T_{F_{\mu}}, E\right)$ is the smallest GFS closed set containing $G_{\delta}$, so, GFS closure of $G_{\delta}$ in $\left(F_{\mu}, T_{F_{\mu}}, E\right)$ is contained in $\operatorname{cl}\left(G_{\delta}\right) \sqcap F_{\mu}$ i.e., $c l_{F_{\mu}}\left(G_{\delta}\right) \subseteq \operatorname{cl}\left(G_{\delta}\right) \sqcap F_{\mu}$.

Conversely,

let $c l_{F_{\mu}}\left(G_{\delta}\right)$ be a GFS closure of $G_{\delta}$ in $\left(F_{\mu}, T_{F_{\mu}}, E\right)$. Since, $c l_{F_{\mu}}\left(G_{\delta}\right)$ is GFS closed set in $\left(F_{\mu}, T_{F_{\mu}}, E\right) \Rightarrow c l_{F_{\mu}}\left(G_{\delta}\right)=$ $K_{\gamma} \sqcap F_{\mu}$ where $K_{\gamma}$ is GFS closed set in $(X, T, E)$. Then, $K_{\gamma}$ is GFS closed set containing $G_{\delta} \Rightarrow \operatorname{cl}\left(G_{\delta}\right) \subseteq K_{\gamma} \Rightarrow$ $\operatorname{cl}\left(G_{\delta}\right) \sqcap F_{\mu} \sqsubseteq K_{\gamma} \sqcap F_{\mu} \sqsubseteq \operatorname{cl}_{F_{\mu}}\left(G_{\delta}\right)$.

Theorem 3.7. Let $(X, T, E)$ be a GFST -space and $G_{\delta} \subseteq F_{\mu} \in G F S(X, E)$. If $H_{v}$ and $K_{\gamma}$ are GFS separated ( respectively, $Q$-separated, strongly separated, weakly separated) in $\left(F_{\mu}, T_{F_{\mu}}, E\right)$, then $H_{v}$ and $K_{\gamma}$ are GFS separated ( respectively, $Q$-separated, strongly separated, weakly separated) in $\left(G_{\delta}, T_{G_{\delta}}, E\right)$.

Proof. As a sample, we will prove the case GFS weakly separated. Let $H_{v}$ and $K_{\gamma}$ be GFS weakly separated sets in $\left(F_{\mu}, T_{F_{\mu}}, E\right)$. Then, $c l_{F_{\mu}}\left(H_{v}\right) \bar{q} K_{\gamma}$ and $H_{v} \bar{q} c l_{F_{\mu}}\left(K_{\gamma}\right)$. Since, $G_{\delta} \sqsubseteq F_{\mu}$. Then, $c l_{G_{\delta}}\left(H_{v}\right)=c l_{F_{\mu}}\left(H_{v}\right) \sqcap G_{\delta} \sqsubseteq c l_{F_{\mu}}\left(H_{v}\right)$ and $c l_{G_{\delta}}\left(K_{\gamma}\right)=c l_{F_{\mu}}\left(K_{\gamma}\right) \sqcap G_{\delta} \subseteq c l_{F_{\mu}}\left(K_{\gamma}\right)$. Therefore, $c l_{G_{\delta}}\left(H_{v}\right) \bar{q} K_{\gamma}$ and $H_{v} \bar{q} c l_{G_{\delta}}\left(K_{\gamma}\right)$. Thus, $H_{v}$ and $K_{\gamma}$ be $G F S$ weakly separated in $\left(G_{\delta}, T_{G_{\delta}}, E\right)$.

Remark 3.10. The converse of Theorem 3.6 is not true in general as shown by the following example:

Example 3.7. Let $X=\left\{x_{1}, x_{2}\right\}, E=\left\{e_{1}, e_{2}\right\}$ and $T^{0}=\left\{\tilde{0}_{\theta}, \tilde{1}_{\Delta}\right\}$ be the GFS indiscrete topology over $(X, E)$.

If $H_{v}=\left\{\left(e_{1}=\left\{\frac{x_{1}}{0.1}, \frac{x_{2}}{0.2}\right\}, 0.1\right)\right\} \sqsubseteq F_{\mu}, K_{\gamma}=\left\{\left(e_{2}=\left\{\frac{x_{1}}{0.1}, \frac{x_{2}}{0.3}\right\}, 0.2\right)\right\} \sqsubseteq F_{\mu}$, where

$F_{\mu}=\left\{\left(e_{1}=\left\{\frac{x_{1}}{0.1}, \frac{x_{2}}{0.2}\right\}, 0.1\right),\left(e_{2}=\left\{\frac{x_{1}}{0.1}, \frac{x_{2}}{0.3}\right\}, 0.2\right)\right\}$. Then, $H_{v}$ and $K_{\gamma}$ are GFS weakly separated sets in $\left(F_{\mu}, T_{F_{\mu}}, E\right)$ but $H_{\nu}$ and $K_{\gamma}$ are not $G F S$ weakly separated sets in $(X, T, E)$.

\section{GENERALIZED FUZZY SOFT CONNECTED SETS IN GENERALIZED FUZZY SOFT TOPOLOGICAL SPACES}

In this section, we introduce different notions of connectedness of GFSSs and study the relation between these notions. Also, we will investegate the characterizations of the generalized fuzzy soft connected sets.

Definition 4.1. A GFSS $F_{\mu}$ in a GFST-space $(X, T, E)$ is called GFS $Q$-connected set if there does not two nonnull GFS $Q$-separated sets $H_{v}$ and $K_{\gamma}$ such that $F_{\mu}=H_{v} \sqcup K_{\gamma}$, Otherwise, $F_{\mu}$ is called not GFS $Q$-connected set.

Definition 4.2. A GFSS $F_{\mu}$ in a GFST-space $(X, T, E)$ is called GFS weakly-connected set if there does not two non-null GFS weakly separated sets $H_{v}$ and $K_{\gamma}$ such that $F_{\mu}=H_{\nu} \sqcup K_{\gamma}$, Otherwise, $F_{\mu}$ is called not GFS weaklyconnected set.

Definition 4.3. A GFSS $F_{\mu}$ in a GFST-space $(X, T, E)$ is called GFS $s$-connected ( respectively, GFS strongly-connected) set if there does not two non-null GFS separated (respectively, not strongly separated) 
sets $H_{v}$ and $K_{\gamma}$ such that $F_{\mu}=H_{v} \sqcup K_{\gamma}$, Otherwise, $F_{\mu}$ is called not GFS $s$-connected ( respectively, GFS strongly-connected) set.

Definition A GFSS $F_{\mu}$ in a $\operatorname{GFST}$-space $(X, T, E)$ is called generalized fuzzy soft clopen set (GFS clopen set, in shoft) if $F_{\mu}, F_{\mu}^{c} \in T$.

Definition 4.4. A GFSS $F_{\mu}$ in a $G F S T$-space $(X, T, E)$ is called $G F S$ clopen-connected set in $(X, E)$ if there does not exist any non-null proper $G F S$ clopen set in $\left(F_{\mu}, T_{F_{\mu}}, E\right)$.

In the above definitions, if we take $\tilde{1}_{\Delta}$ instead of $F_{\mu}$, then the $G F S T$-space $(X, T, E)$ is called GFS $Q-$ connected (respectively, GFS weakly-connected, GFS s-connected, GFS strongly-connected, GFS clopen-connected ) space.

Theorem 4.1. The GFS -weakly connected set in $(X, E)$ is a GFS $Q$-connected.

Proof. Let $F_{\mu}$ be a GFS -weakly connected set in $(X, E)$. Suppose $F_{\mu}$ is not a GFS $Q$-connected. Then, there exist two non-null GFS $Q$-separated sets $H_{v}$ and $K_{\gamma}$ such that $F_{\mu}=H_{v} \sqcup K_{\gamma}$. By Remark $3.1, H_{v}$ and $K_{\gamma}$ are non-null GFS weakly separated sets in $(X, E)$ such that $F_{\mu}=H_{v} \sqcup K_{\gamma}$. Therefore, $F_{\mu}$ is not a GFS-weakly connected set in $(X, E)$, a contradiction. Hence, $F_{\mu}$ is a GFS $Q$-connected.

Remark 4.1. A GFS Q -connected set may not be GFS weakly-connected as shown by the following example.

Example 4.1. Let $X=\left\{x_{1}, x_{2}\right\}, E=\left\{e_{1}, e_{2}\right\}$ and $T=\left\{\tilde{0}_{\theta}, \tilde{1}_{\Delta},\left\{\left(e_{1}=\left\{\frac{x_{1}}{0.3}, \frac{x_{2}}{0.2}\right\}, 0.3\right),\left(e_{2}=\left\{\frac{x_{1}}{0.5}, \frac{x_{2}}{0.3}\right\}, 0.4\right)\right\}\right\}$ be a GFS topology over $(X, E)$. Let $F_{\mu}=\left\{\left(e_{1}=\left\{\frac{x_{1}}{0.1}, \frac{x_{2}}{0.1}\right\}, 0.3\right)\right\}$. Then there exist $H_{v}=\left\{\left(e_{1}=\left\{\frac{x_{1}}{0.1}\right\}, 0.2\right)\right\}$ and $K_{\gamma}=$ $\left\{\left(e_{1}=\left\{\frac{x_{2}}{0.1}\right\}, 0.3\right)\right\}$ such that $\operatorname{cl}\left(H_{v}\right) \bar{q} K_{\gamma}$ and $H_{v} \bar{q} c l\left(K_{\gamma}\right), F_{\mu}=H_{v} \sqcup K_{\gamma}$. So, $F_{\mu}$ is not a $G F S$ weakly-connected. If we take $M_{\psi}=\left\{\left(e_{1}=\left\{\frac{x_{1}}{0.1}, \frac{x_{2}}{\beta}\right\}, \lambda\right)\right\}, N_{\eta}=\left\{\left(e_{1}=\left\{\frac{x_{1}}{\alpha}, \frac{x_{2}}{0.1}\right\}, 0.3\right)\right\}$ where $\alpha, \beta \leq 0.1$ and $\lambda \leq 0.3$. Then $\operatorname{cl}\left(M_{\psi}\right) \sqcap N_{\eta} \neq \tilde{0}_{\theta}$ and $M_{\psi} \sqcap \operatorname{cl}\left(N_{\eta}\right) \neq \tilde{0}_{\theta}$. Therefore, $M_{\psi}$ and $N_{\eta}$ are not GFS $Q$ separated sets. Hence, $F_{\mu}$ is a GFS $Q-$ connected.

Theorem 4.2. A GFSC $_{1}$-connected set in $(X, E)$ is $G F S$ weakly-connected.

Proof. Let $F_{\mu}$ be a $G F S C_{1}$-connected set in $(X, E)$. Suppose $F_{\mu}$ is not $G F S$ weakly-connected. Then, there exist two non-null GFS weakly separated sets $H_{v}$ and $K_{\gamma}$ such that $F_{\mu}=H_{v} \sqcup K_{\gamma}$. By Theorem 3.3, there exist GFS open sets $M_{\psi}$ and $N_{\eta}$ such that $H_{v} \sqsubseteq M_{\psi}, K_{\gamma} \sqsubseteq N_{\eta}, H_{\nu} \bar{q} N_{\eta}$ and $M_{\psi} \bar{q} K_{\gamma}$. Then, $F_{\mu} \sqsubseteq M_{\psi} \sqcup N_{\eta}$. Also, $F_{\mu} \sqcap M_{\psi} \neq \tilde{0}_{\theta}$. For, if $F_{\mu} \sqcap M_{\psi}=\tilde{0}_{\theta}$, then $F_{\mu} \sqcap H_{v}=\tilde{0}_{\theta}$ so that $H_{v}=\tilde{0}_{\theta}$ (since $F_{\mu}=H_{v} \sqcup K_{\gamma}$ implies that $H_{v} \sqsubseteq F_{\mu}$ ), which contradiction that $H_{v}$ is a non-null. Similarly, $F_{\mu} \sqcap N_{\eta} \neq \tilde{0}_{\theta}$.

Also, $M_{\psi} \sqcap N_{\eta} \sqsubseteq\left(F_{\mu}\right)^{c}$. For, if $M_{\psi} \sqcap N_{\eta} \nsubseteq F_{\mu}^{c}$, then there exist $x \in X, e \in E$ such that

$M(e)(x)>1-F(e)(x), \psi(e)>1-\mu(e)$ and $N(e)(x)>1-F(e)(x), \eta(e)>1-\mu(e)$

This means $M(e)(x)+F(e)(x)>1, \psi(e)+\mu(e)>1$ and $N(e)(x)+F(e)(x)>1, \eta(e)+\mu(e)>1$. Since, $F_{\mu}=$ $H_{v} \sqcup K_{\gamma}$, then $M(e)(x)+H(e)(x)>1, \psi(e)+v(e)>1$ or $M(e)(x)+K(e)(x)>1, \psi(e)+\gamma(e)>1$ and

$N(e)(x)+H(e)(x)>1, \eta(e)+v(e)>1$ or $N(e)(x)+K(e)(x)>1, \eta(e)+\gamma(e)>1$. Hence, $\left(M_{\psi} q H_{v}\right.$ or $\left.M_{\psi} q K_{\gamma}\right)$ and $\left(N_{\eta} q H_{v}\right.$ or $\left.N_{\eta} q K_{\gamma}\right)$. This a contradiction. So, $F_{\mu}$ is a GFS weakly-connected.

Remark 4.2. The GFS weakly-connected set may not be a $G F S C_{1}$-connected as shown by the following example.

Example 4.2. Let $X=\left\{x_{1}, x_{2}\right\}, E=\left\{e_{1}\right\}$ and $T=\left\{\tilde{0}_{\theta}, \tilde{1}_{\Delta},\left\{\left(e_{1}=\left\{\frac{x_{1}}{0.7}, \frac{x_{2}}{0.8}\right\}, 0.6\right)\right\},\left\{\left(e_{1}=\left\{\frac{x_{1}}{0.2}, \frac{x_{2}}{0.3}\right\}, 0.1\right)\right\}\right\}$ be a GFS topology over $(X, E)$ and $F_{\mu}=\left\{\left(e_{1}=\left\{\frac{x_{1}}{0.4}, \frac{x_{2}}{0.4}\right\}, 0.5\right)\right\}$. Then, there exist two $G F S$ open sets $H_{v}=\left\{\left(e_{1}=\right.\right.$ 
$\left.\left.\left\{\frac{x_{1}, x_{2}}{0.7^{\prime} 0.8}\right\}, 0.6\right)\right\}$ and $K_{\gamma}=\left\{\left(e_{1}=\left\{\frac{x_{1}}{0.2_{2} \cdot x_{2}}\right\}, 0.1\right)\right\}$ such that $F_{\mu} \subseteq H_{v} \sqcup K_{\gamma}, H_{v} \sqcap K_{\gamma} \sqsubseteq F_{\mu}^{c}, F_{\mu} \sqcap H_{v} \neq \tilde{0}_{\theta}$ and $F_{\mu} \sqcap K_{\gamma} \neq \tilde{0}_{\theta}$. So, $F_{\mu}$ is not a $G F S C_{1}$-connected. If we take $M_{\psi}=\left\{\left(e_{1}=\left\{\frac{x_{1}}{0.4}, \frac{x_{2}}{\beta}\right\}, \lambda\right)\right\}, N_{\eta}=\left\{\left(e_{1}=\left\{\frac{x_{1}}{\alpha}, \frac{x_{2}}{0.4}\right\}, 0.5\right)\right\}$ where $\alpha, \beta \leq$ 0.4 and $\lambda \leq 0.5$. Then $\quad \operatorname{cl}\left(M_{\psi}\right) q N_{\eta}$ and $M_{\psi} q c l\left(N_{\eta}\right)$. Therefore, $M_{\psi}$ and $N_{\eta}$ are not GFS weakly separated sets. Hence, $F_{\mu}$ is a GFS weakly-connected.

Theorem 4.3. A GFS weakly-connected set in $(X, E)$ is $G F S C_{2}$-connected.

Proof. Let $F_{\mu}$ be a GFS weakly-connected set in $(X, E)$. Suppose $F_{\mu}$ is not $G F S C_{2}$-connected. Then, there exist $H_{v}$ and $K_{\gamma} \in T$ such that $F_{\mu} \sqsubseteq H_{v} \sqcup K_{\gamma}, F_{\mu} \sqcap H_{v} \sqcap K_{\gamma}=\tilde{0}_{\theta}, F_{\mu} \sqcap H_{v} \neq \tilde{0}_{\theta}$ and $F_{\mu} \sqcap K_{\gamma} \neq \tilde{0}_{\theta}$. Then, $F_{\mu}=M_{\psi} \sqcup$ $N_{\eta}$ where $M_{\psi}=F_{\mu} \sqcap H_{v} \sqsubseteq H_{v}$ and $N_{\eta}=F_{\mu} \sqcap K_{\gamma} \sqsubseteq K_{\gamma}$. Since $F_{\mu} \sqcap H_{v} \sqcap K_{\gamma}=\tilde{0}_{\theta}$ and $M_{\psi} \sqsubseteq H_{v}$, then $F_{\mu} \sqcap M_{\psi} \sqcap$ $K_{\gamma}=\tilde{0}_{\theta}$. Also, since $M_{\psi} \sqsubseteq F_{\mu}$, then $M_{\psi} \sqcap K_{\gamma}=\tilde{0}_{\theta}$. Therefore, $M_{\psi} \bar{q} K_{\gamma}$, Similarly, $N_{\eta} \bar{q} H_{v}$. Hence, $F_{\mu}$ is not a GFS weakly-connected. This complete the proof.

Theorem 4.4. A GFS weakly-connected set in $(X, E)$ is $G F S C_{3}$-connected.

Proof. Let $F_{\mu}$ be a The GFS weakly-connected set in $(X, E)$. Suppose $F_{\mu}$ is not $G F S C_{3}$-connected. Then, there exist $H_{v}$ and $K_{\gamma} \in T$ such that $F_{\mu} \sqsubseteq H_{v} \sqcup K_{\gamma}, H_{v} \sqcap K_{\gamma} \sqsubseteq F_{\mu}^{c}, H_{v} \nsubseteq F_{\mu}^{c}$ and $K_{\gamma} \nsubseteq F_{\mu}^{c}$. Then, $F_{\mu}=M_{\psi} \sqcup N_{\eta}$ where $M_{\psi}=F_{\mu} \sqcap H_{v} \sqsubseteq H_{v}$ and $N_{\eta}=F_{\mu} \sqcap K_{\gamma} \sqsubseteq K_{\gamma}$. Let $J_{\sigma}$ and $L_{\rho} \in G F S(X, E)$ defined by:

$J_{\sigma}= \begin{cases}M_{\psi}, & H_{v} \sqsupseteq K_{\gamma}, \\ \tilde{0}_{\theta}, & \text { otherwise }\end{cases}$

$L_{\rho}= \begin{cases}N_{\eta}, & K_{\gamma} \sqsupset H_{v}, \\ \tilde{0}_{\theta}, & \text { otherwise }\end{cases}$

Then $F_{\mu}=J_{\sigma} \sqcup L_{\rho}$.

Now, $J(e)(x) \neq 0, \sigma(e) \neq 0$. For, $J(e)(x)=0, \sigma(e)=0$. Since, $H_{v} \nsubseteq F_{\mu}^{c}$, then there exist $x \in X, e \in E$ such that $H(e)(x)+F(e)(x)>1, v(e)+\mu(e)>1$. Then, $H(e)(x)>K(e)(x), v(e)>\gamma(e)$. For, $H(e)(x) \leq K(e)(x), v(e) \leq$ $\gamma(e)$ implies $K(e)(x)+F(e)(x)>1, \gamma(e)+\mu(e)>1$ and hence $\left(H_{v} \sqcap K_{\gamma}\right)(e)(x)>1-F_{\mu}(e)(x)$ i.e., $H(e)(x)>$ $1-F(e)(x), v(e)>1-\mu(e)$ and $K(e)(x)>1-F(e)(x), \gamma(e)>1-\mu(e)$ this is a contradiction with $H_{v} \sqcap K_{\gamma}$ ㄷ $F_{\mu}^{c}$. So, $J(e)(x) \neq 0, \sigma(e) \neq 0$. Similarly, $L(e)(x) \neq 0, \rho(e) \neq 0$. Also, $J_{\sigma} \subseteq M_{\psi} \sqsubseteq H_{v}$ and $L_{\rho} \subseteq N_{\eta} \subseteq K_{\gamma}$. Now, $J_{\sigma} \bar{q} K_{\gamma}$. For, if $J_{\sigma} q K_{\gamma}$, then there exist $x \in X, e \in E$ such that $J(e)(x)+K(e)(x)>1, \sigma(e)+\gamma(e)>1$ and hence $J(e)(x)>0, \sigma(e)>0$. This means $H(e)(x) \geq K(e)(x), v(e) \leq \gamma(e)$ and so $F(e)(x)=M(e)(x), \mu(e)=\psi(e)$ implying $F(e)(x)+H(e)(x)>1, \mu(e)+v(e)>1$ and thus $\left(H_{v} \sqcap K_{\gamma}\right)(e)(x)>1-F_{\mu}(e)(x)$ which is a contradiction with $H_{v} \sqcap K_{\gamma} \subseteq F_{\mu}^{c}$. Similarly, $L_{\rho} \bar{q} H_{v}$. Thus, $J_{\sigma}$ and $L_{\rho}$ are GFS weakly separated and $F_{\mu}=J_{\sigma} \sqcup L_{\rho}$. So, $F_{\mu}$ is not a GFS weakly-connected. This a contradiction. Then $F_{\mu}$ is a $G F S C_{3}$-connected.

Remark 4.3. The $\mathrm{GFSC}_{3}$-connected set (respectively, GFSC $C_{2}$-connected) may not be a GFS weakly-connected as shown by the following example.

Example 4.3. Let $X=\left\{x_{1}, x_{2}\right\}, E=\left\{e_{1}\right\}$ and

$T=\left\{\tilde{0}_{\theta}, \tilde{1}_{\Delta},\left\{\left(e_{1}=\left\{\frac{x_{1}}{2 / 3}, \frac{x_{2}}{1 / 3}\right\}, 1 / 3\right)\right\},\left\{\left(e_{1}=\left\{\frac{x_{1}}{1 / 3}, \frac{x_{2}}{2 / 3}\right\}, 2 / 3\right)\right\},\left\{\left(e_{1}=\left\{\frac{x_{1}}{1 / 3}, \frac{x_{2}}{1 / 3}\right\}, 1 / 3\right)\right\},\left\{\left(e_{1}=\left\{\frac{x_{1}}{2 / 3}, \frac{x_{2}}{2 / 3}\right\}, 2 / 3\right)\right\}\right\}$ be a GFS

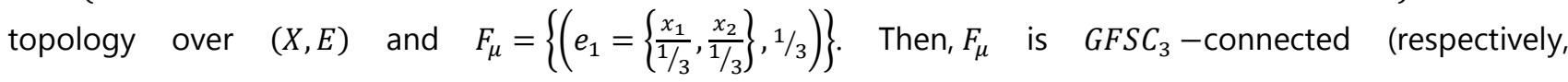
$G_{F S C_{2}}$-connected). But $F_{\mu}$ is not a GFS weakly-connected as there exist GFS weakly separated sets $H_{v}=$ $\left\{\left(e_{1}=\left\{\frac{x_{1}}{1 / 3}\right\}, 1 / 3\right)\right\}, K_{\gamma}=\left\{\left(e_{1}=\left\{\frac{x_{2}}{1 / 3}\right\}, 1 / 3\right)\right\}$ such that $F_{\mu}=H_{v} \sqcup K_{\gamma}$.

Theorem 4.5. The $\mathrm{GFSC}_{3}$-connected set in $(X, E)$ is a GFS Q-connected. 
Proof. Let $F_{\mu}$ be a $G F S C_{3}$-connected set in $(X, E)$. Suppose $F_{\mu}$ is not GFS $Q$-connected. Then, there exist two non-null GFS $Q$-separated sets $H_{v}$ and $K_{\gamma}$ such that $F_{\mu}=H_{v} \sqcup K_{\gamma}, \operatorname{cl}\left(H_{v}\right) \sqcap K_{\gamma}=H_{v} \sqcap \operatorname{cl}\left(K_{\gamma}\right)=\tilde{0}_{\theta}$. This implies that $K_{\gamma} \subseteq\left[\operatorname{cl}\left(H_{v}\right)\right]^{c}$ and $H_{v} \subseteq\left[\operatorname{cl}\left(K_{\gamma}\right)\right]^{c}$. Let $M_{\psi}=\left[\operatorname{cl}\left(H_{v}\right)\right]^{c}$ and $N_{\eta}=\left[\operatorname{cl}\left(K_{\gamma}\right)\right]^{c}$. Then, $M_{\psi}$ and $N_{\eta}$ are non- null GFS open sets such that $F_{\mu} \sqsubseteq M_{\psi} \sqcup N_{\eta}$. Now, $M_{\psi} \sqcap N_{\eta}=\left[\operatorname{cl}\left(H_{v}\right)\right]^{c} \sqcap\left[\operatorname{cl}\left(K_{\gamma}\right)\right]^{c}=\left[\operatorname{cl}\left(H_{v}\right) \sqcup \operatorname{cl}\left(K_{\gamma}\right)\right]^{c}=$ $\left[\operatorname{cl}\left(H_{v} \sqcup K_{\gamma}\right)\right]^{c} \sqsubseteq F_{\mu}^{c}$. Aso, $M_{\psi} \nsubseteq F_{\mu}^{c}$. For, if $M_{\psi} \subseteq F_{\mu}^{c}$, then $F_{\mu} \subseteq M_{\psi}^{c}=\operatorname{cl}\left(H_{v}\right)$ which would imply $K_{\gamma}=\tilde{0}_{\theta}$ ( since $\left.\operatorname{cl}\left(H_{v}\right) \sqcap K_{\gamma}=\tilde{0}_{\theta}\right)$. This is a contradiction. Similarly, $N_{\eta} \nsubseteq F_{\mu}^{c}$. Therefore, $F_{\mu}$ is not $G F S C_{3}-$ connected. So, $F_{\mu}$ is GFS $Q$-connected.

Remark 4.4. A GFS Q-connected set may not be $\mathrm{GFSC}_{3}$-connected as shown by the following example.

Example 4.4. Let $X=\left\{x_{1}, x_{2}\right\}, E=\left\{e_{1}\right\}$ and

$T=\left\{\tilde{0}_{\theta}, \tilde{1}_{\Delta},\left\{\left(e_{1}=\left\{\frac{x_{1}}{0.6^{\prime}}, \frac{x_{2}}{0.2}\right\}, 0.3\right)\right\},\left\{\left(e_{1}=\left\{\frac{x_{1}}{0.2}, \frac{x_{2}}{0.7}\right\}, 0.4\right)\right\},\left\{\left(e_{1}=\left\{\frac{x_{1}}{0.6}, \frac{x_{2}}{0.7}\right\}, 0.4\right)\right\},\left\{\left(e_{1}=\left\{\frac{x_{1}}{0.2}, \frac{x_{2}}{0.2}\right\}, 0.3\right)\right\}\right\}, \quad$ be $\quad$ a $\quad$ GFS topology over $(X, E)$ and $F_{\mu}=\left\{\left(e_{1}=\left\{\frac{x_{1}}{0.6^{\prime} 0.7}\right\}, 0.4\right)\right\}$.

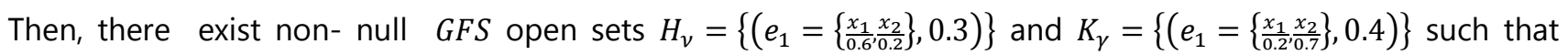
$F_{\mu} \sqsubseteq H_{v} \sqcup K_{\gamma}, H_{v} \sqcap K_{\gamma} \sqsubseteq F_{\mu}^{c}, H_{v} \nsubseteq F_{\mu}^{c}$ and $K_{\gamma} \nsubseteq F_{\mu}^{c}$. So, $F_{\mu}$ is not $G F S C_{3}$-connected. However, $F_{\mu}$ is GFS $Q$-connected.

Theorem 4.6. A GFSS $F_{\mu}$ in $(X, E)$ is $G F S C_{2}$-connected if and only if $F_{\mu}$ is GFS $s$-connected.

Proof. Let $F_{\mu}$ be a $G F S C_{2}$-connected set in $(X, E)$. Suppose $F_{\mu}$ is not a $G F S s$-connected. Then there exist non-null GFS separated sets $H_{v}$ and $K_{\gamma}$ in $(X, E)$ such that $F_{\mu}=H_{v} \sqcup K_{\gamma}$. Then, there exist two non- null GFS open sets $M_{\psi}$ and $N_{\eta}$ such that $H_{v} \sqsubseteq M_{\psi}, K_{\gamma} \sqsubseteq N_{\eta}$, and $H_{v} \sqcap N_{\eta}=K_{\gamma} \sqcap M_{\psi}=\tilde{0}_{\theta}$. Then, $F_{\mu} \sqsubseteq M_{\psi} \sqcup N_{\eta}$.

Now, $\quad F_{\mu} \sqcap M_{\psi} \sqcap N_{\eta}=\left(H_{v} \sqcup K_{\gamma}\right) \sqcap M_{\psi} \sqcap N_{\eta}=\left(H_{v} \sqcap M_{\psi} \sqcap N_{\eta}\right) \sqcup\left(K_{\gamma} \sqcap M_{\psi} \sqcap N_{\eta}\right)=\tilde{0}_{\theta} \quad$ and $\quad F_{\mu} \sqcap M_{\psi}=\left(H_{v} \sqcup\right.$ $\left.K_{\gamma}\right) \sqcap M_{\psi}=\left(H_{v} \sqcap M_{\psi}\right) \sqcup\left(K_{\gamma} \sqcap M_{\psi}\right)=H_{v} \neq \tilde{0}_{\theta}$. Similarly, $F_{\mu} \sqcap N_{\eta} \neq \tilde{0}_{\theta}$. So, $F_{\mu}$ is not $G_{F S C}$-connected which is a contradiction.

Conversely, let $F_{\mu}$ be GFS $s$-connected. Suppose that $F_{\mu}$ is not $G F S C_{2}$-connected. Then there exist two nonnull GFS open sets $M_{\psi}$ and $N_{\eta}$ such that $F_{\mu} \subseteq M_{\psi} \sqcup N_{\eta}, F_{\mu} \sqcap M_{\psi} \sqcap N_{\eta}=\tilde{0}_{\theta}, F_{\mu} \sqcap M_{\psi} \neq \tilde{0}_{\theta}, F_{\mu} \sqcap N_{\eta} \neq \tilde{0}_{\theta}$. Hence, $F_{\mu}=H_{v} \sqcup K_{\gamma}$ where $H_{v}=F_{\mu} \sqcap M_{\psi} \sqsubseteq M_{\psi}$ and $K_{\gamma}=F_{\mu} \sqcap N_{\eta} \sqsubseteq N_{\eta}$. Also, $K_{\gamma} \sqcap M_{\psi}=\left(F_{\mu} \sqcap N_{\eta}\right) \sqcap M_{\psi}=\tilde{0}_{\theta}$, Similarly, $H_{v} \sqcap N_{\eta}=\tilde{0}_{\theta}$. So, $F_{\mu}$ is not GFS $s$-connected and this complete the proof.

Theorem 4.7. The $\mathrm{GFSC}_{4}$-connected set in $(X, E)$ is a GFS strongly-connected.

Proof. Let $F_{\mu}$ be a $G F S C_{4}$-connected set in $(X, E)$. Suppose $F_{\mu}$ is not a $G F S$ strongly-connected. Then there exist two non-null GFS strongly separated sets $H_{v}$ and $K_{\gamma}$ in $(X, E)$ such that $F_{\mu}=H_{v} \sqcup K_{\gamma}$. So, there exist two non- null GFS open sets $M_{\psi}$ and $N_{\eta}$ such that

$H_{v} \sqsubseteq M_{\psi}, K_{\gamma} \sqsubseteq N_{\eta}$, and $H_{v} \sqcap N_{\eta}=K_{\gamma} \sqcap M_{\psi}=\tilde{0}_{\theta}$,

$H_{v}$ and $M_{\psi} G F S$ quasi-coincident with respect to $H_{v}$, and $K_{\gamma}$ and $N_{\eta} G F S$ quasi-coincident with respect to $K_{\gamma}$.

Then, for every $x, e \in S\left(H_{v}\right)$ we have $H(e)(x)+M(e)(x)>1$ and $v(e)+\psi(e)>1$ and for every $x, e \in S\left(K_{\gamma}\right)$ we have $K(e)(x)+N(e)(x)>1$ and $\gamma(e)+\eta(e)>1$. Then, $F_{\mu} \sqsubseteq M_{\psi} \sqcup N_{\eta}$. Also, $F_{\mu} \sqcap M_{\psi} \sqcap N_{\eta}=\tilde{0}_{\theta}$.

Again, $F(e)(x)+M(e)(x)>H(e)(x)+M(e)(x)$ and $\mu(e)+\psi(e)>v(e)+\psi(e)>$ for every $x, e \in S\left(H_{v}\right)$. Therefore, $M_{\psi} \nsubseteq F_{\mu}^{c}$, Similarly, $N_{\eta} \nsubseteq F_{\mu}^{c}$. Thus, $F_{\mu}$ is not a $G F S C_{4}$-connected. This is a contradiction. So, $F_{\mu}$ is a GFS strongly-connected. 
Remark 4.5. A GFS strongly-connected set may not be $G F S C_{4}$-connected as shown by the following example.

Example 4.5. Let $X=\left\{x_{1}, x_{2}, x_{3}\right\}, E=\left\{e_{1}, e_{2}\right\}$ and $T=\left\{\tilde{0}_{\theta}, \tilde{1}_{\Delta},\left\{\left(e_{1}=\left\{\frac{x_{1}}{0.7}\right\}, 0.9\right)\right\},\left\{\left(e_{2}=\left\{\frac{x_{2}}{0.7} \frac{x_{3}}{0.8}\right\}, 0.6\right)\right\},\left\{\left(e_{1}=\left\{\frac{x_{1}}{0.7}\right\}, 0.9\right),\left(e_{2}=\left\{\frac{x_{2}}{0.7} \frac{x_{3}}{0.8}\right\}, 0.6\right)\right\}\right\}$, be a GFS topology over $(X, E)$.

Let $F_{\mu}=\left\{\left(e_{1}=\left\{\frac{x_{1}}{0.7}\right\}, 0.9\right),\left(e_{2}=\left\{\frac{x_{2}}{0.7}, \frac{x_{3}}{0.8}\right\}, 0.6\right)\right\}$ and $H_{v}=\left\{\left(e_{1}=\left\{\frac{x_{1}}{0.7}\right\}, 0.9\right)\right\}, K_{\gamma}=\left\{\left(e_{2}=\left\{\frac{x_{2}}{0.7} \frac{x_{3}}{0.8}\right\}, 0.6\right)\right\} \in T$.

Then, $F_{\mu} \sqsubseteq H_{v} \sqcup K_{\gamma}, F_{\mu} \sqcap H_{v} \sqcap K_{\gamma}=\tilde{0}_{\theta}, H_{v} \nsubseteq F_{\mu}^{c}$ and $K_{\gamma} \nsubseteq F_{\mu}^{c}$. So, $F_{\mu}$ is not a $G F S C_{4}$-connected. However, $F_{\mu}$ is GFS strongly-connected.

Remark 4.6. A GFS Q-connected set and GFS strongly-connected are independent concepts as shown by the following examples.

Example 4.6. Let $X=\left\{x_{1}, x_{2}, x_{3}\right\}, E=\left\{e_{1}, e_{2}\right\}$ and

$T=\left\{\tilde{0}_{\theta}, \tilde{1}_{\Delta},\left\{\left(e_{1}=\left\{\frac{x_{1}}{0.8}\right\}, 0.9\right)\right\},\left\{\left(e_{2}=\left\{\frac{x_{2}}{0.9} \frac{x_{3}}{0.9}\right\}, 0.7\right)\right\},\left\{\left(e_{1}=\left\{\frac{x_{1}}{0.8}\right\}, 0.9\right),\left(e_{2}=\left\{\frac{x_{2}}{0.9}, \frac{x_{3}}{0.9}\right\}, 0.7\right)\right\}\right\}$ be a GFS topology over $(X, E)$. Let $F_{\mu}=\left\{\left(e_{1}=\left\{\frac{x_{1}}{0.6}\right\}, 0.7\right),\left(e_{2}=\left\{\frac{x_{2}}{0.7}, \frac{x_{3}}{0.8}\right\}, 0.6\right)\right\}$.

Then, there exist two non-null GFS strongly separated $H_{v}=\left\{\left(e_{1}=\left\{\frac{x_{1}}{0.6}, 0.7\right)\right\}\right.$ and $K_{\gamma}=\left\{\left(e_{2}=\left\{\frac{x_{2}}{0.7}, \frac{x_{3}}{0.8}, 0.6\right)\right\}\right.$ such that $F_{\mu}=H_{v} \sqcup K_{\gamma}$. So, $F_{\mu}$ is not GFS strongly-connected. However, $F_{\mu}$ is GFS $Q$-connected as $\operatorname{cl}\left(H_{v}\right) \Pi$ $K_{\gamma} \neq \tilde{0}_{\theta}$ and also $H_{v} \sqcap \operatorname{cl}\left(K_{\gamma}\right) \neq \tilde{0}_{\theta}$.

Example 4.7. Let $X=\left\{x_{1}, x_{2}\right\}, E=\left\{e_{1}, e_{2}\right\}$ and $T=\left\{\tilde{0}_{\theta}, \tilde{1}_{\Delta},\left\{\left(e_{1}=\left\{\frac{x_{1}}{0.4}\right\}, 0.4\right),\left(e_{2}=\left\{\frac{x_{1}}{1}, \frac{x_{2}}{1}\right\}, 1\right)\right\},\left\{\left(e_{1}=\left\{\frac{x_{1}}{1}, \frac{x_{2}}{1}\right\}, 1\right),\left(e_{2}=\left\{\frac{x_{2}}{0.4}\right\}, 0.4\right)\right\},\left\{\left(e_{1}=\left\{\frac{x_{1}}{0.4}\right\}, 0.4\right),\left(e_{2}=\right.\right.\right.$ $\left.\left.\left.\left\{\frac{x_{2}}{0.4}\right\}, 0.4\right)\right\}\right\}$ be a GFS topology over $(X, E)$. Let $F_{\mu}=\left\{\left(e_{1}=\left\{\frac{x_{1}}{0.4}\right\}, 0.4\right),\left(e_{2}=\left\{\frac{x_{2}}{0.4}\right\}, 0.4\right)\right\}$. Then, there exist non- null GFS $Q$-separated sets $H_{v}=\left\{\left(\left(e_{1}=\left\{\frac{x_{1}}{0.4}\right\}, 0.4\right)\right)\right\}$ and $K_{\gamma}=\left\{\left(e_{2}=\left\{\frac{x_{2}}{0.4}\right\}, 0.4\right)\right\}$ such that $F_{\mu}=H_{v} \sqcup K_{\gamma}$. So, $F_{\mu}$ is not GFS $Q$-connected. However, $F_{\mu}$ is GFS strongly-connected as $H_{v}$ and $K_{\gamma}$ are not GFS strongly separated.

Remark 4.7. A $\mathrm{GFSC}_{2}$-connected set may not be GFS $Q$-connected as shown by the following example.

Example 4.8. Let $X=\left\{x_{1}, x_{2}\right\}, E=\left\{e_{1}, e_{2}\right\}$ and

$T=\left\{\tilde{0}_{\theta}, \tilde{1}_{\Delta},\left\{\left(e_{1}=\left\{\frac{x_{1}}{1 / 3}, \frac{x_{2}}{1}\right\}, 1 / 3\right),\left(e_{2}=\left\{\frac{x_{1}}{1}, \frac{x_{2}}{1}\right\}, 1\right)\right\},\left\{\left(e_{1}=\left\{\frac{x_{1}}{1}, \frac{x_{2}}{1}\right\}, 1\right),\left(e_{2}=\left\{\frac{x_{1}}{1}, \frac{x_{2}}{1 / 3}\right\}, 1 / 3\right)\right\}\right.$,

$\left.\left\{\left(e_{1}=\left\{\frac{x_{1}}{1 / 3}, \frac{x_{2}}{1}\right\}, 1 / 3\right),\left(e_{2}=\left\{\frac{x_{1}}{1}, \frac{x_{2}}{1 / 3}\right\}, 1 / 3\right)\right\}\right\}$ be a GFS topology over $(X, E)$.

Let $F_{\mu}=\left\{\left(e_{1}=\left\{\frac{x_{1}}{2 / 3}\right\}, 2 / 3\right),\left(e_{2}=\left\{\frac{x_{2}}{2 / 3}\right\}, 2 / 3\right)\right\}$. Then, $F_{\mu}$ can be expressed as union of two non-null GFS $Q$-separated sets $H_{v}=\left\{\left(e_{1}=\left\{\frac{x_{1}}{2 / 3}\right\}, 1 / 3\right)\right\}$ and $K_{\gamma}=\left\{\left(e_{2}=\left\{\frac{x_{2}}{2 / 3}\right\}, 2 / 3\right)\right\}$. So, $F_{\mu}$ is not a GFS $Q$-connected. However, $F_{\mu}$ is a $G F S C_{2}$-connected as if we take

$M_{\psi}=\left\{\left(e_{1}=\left\{\frac{x_{1}}{1 / 3}, \frac{x_{2}}{1}\right\}, 1 / 3\right),\left(e_{2}=\left\{\frac{x_{1}}{1}, \frac{x_{2}}{1}\right\}, 1\right)\right\}$ and $N_{\eta}=\left\{\left(e_{1}=\left\{\frac{x_{1}}{1}, \frac{x_{2}}{1}\right\}, 1\right),\left(e_{2}=\left\{\frac{x_{1}}{1}, \frac{x_{2}}{1 / 3}\right\}, 1 / 3\right)\right\} \in T$, then $F_{\mu}$ ㄷ $M_{\psi} \sqcup N_{\eta}$, but $F_{\mu} \sqcap M_{\psi} \sqcap N_{\eta} \neq \tilde{0}_{\theta}$. 
Remark 4.8. A GFS clopen-connected set may not be a GFS $s$-connected (respectively, GFS strongly-connected, GFS $Q$-connected, GFS weakly-connected, $G F S C_{i}$-connected for $\left.i=1,2,3,4\right)$. In fact, $F_{\mu}$ defined in Example 4.6 is a GFS clopen-connected, but it is not a GFS strongly-connected set and in Example 4.8 is a GFS clopen-connected, but it is not a GFS $Q$-connected set. Therefore, it is not a GFS $s$-connected, not a GFS weakly-connected set and not a $G F S C_{i}$-connected set for $i=1,2,3,4$.

Remark 4.9. A GFS $s$-connected (respectively, GFS strongly-connected, GFS $Q$-connected, GFS weakly-connected, $G F S C_{i}$-connected for $\left.i=1,2,3,4\right)$ set may not be $G F S$ clopen-connected as shown by the following example.

Example 4.9. Let $X=\left\{x_{1}, x_{2}\right\}, E=\left\{e_{1}\right\}$ and $T=\left\{\tilde{0}_{\theta}, \tilde{1}_{\Delta},\left\{\left(e_{1}=\left\{\frac{x_{1}}{0.3}\right\}, 0.3\right)\right\},\left\{\left(e_{1}=\left\{\frac{x_{1}}{0.5}, \frac{x_{2}}{0.6}\right\}, 0.5\right)\right\}\right\}$ be a GFS topology over $(X, E)$. Let $F_{\mu}=\left\{\left(e_{1}=\left\{\frac{x_{1}}{0.7}\right\}, 0.7\right)\right\}$. Then, $F_{\mu}$ is a GFS $s$-connected, GFS strongly-connected, GFS $Q$-connected, GFS weakly-connected, GFSC $C_{i}$-connected for $\left.i=1,2,3,4\right)$. But since $\left\{\left(e_{1}=\left\{\frac{x_{1}}{0.5}\right\}, 0.5\right)\right\}$ is a non-null proper clopen GFSS in $F_{\mu}$. So, $F_{\mu}$ is not a GFS clopen-connected.

Remark 4.10. In a GFST-space $(X, T, E)$. The classes of $G F S$-connected, GFS strongly-connected, GFS $Q$-connected, GFS weakly-connected, GFSC $C_{i}$-connected for $i=1,2,3,4$, can be discribed by the following diagram.

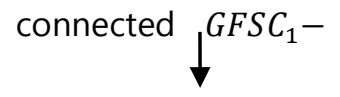

weakly-connectedGFS

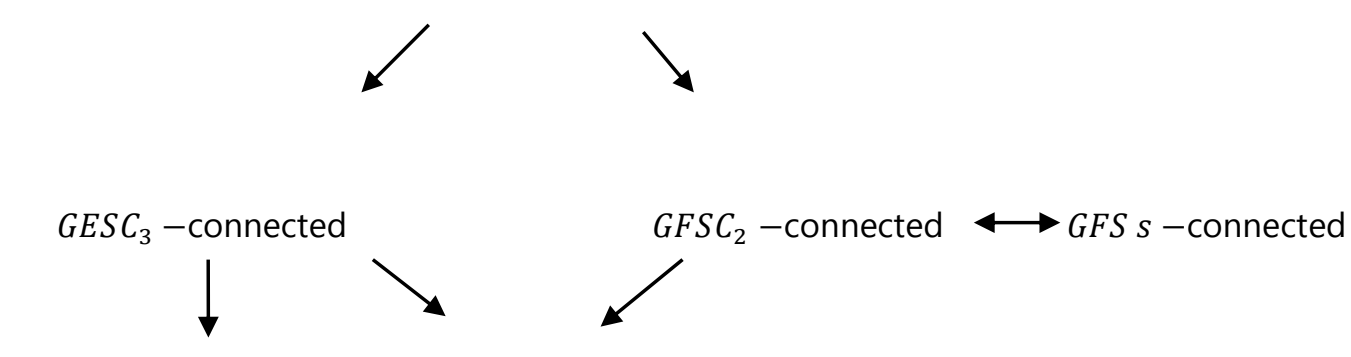

connected $\quad \mathrm{GFSC}_{4}$-connected $\quad$ GFS Q -

strongly-connected GFS

Theorem 4.8. Let $\left(X, T_{1}, E\right)$ and $\left(Y, T_{2}, K\right)$ be a GFST-spaces and $f_{\text {up }}:\left(X, T_{1}, E\right) \rightarrow\left(Y, T_{1}, K\right)$ be a GFScontinuous bijective mapping. If $F_{\mu}$ is a $G F S C_{i}$-connected (respectively, GFS $s$-connected, GFS strongly-connected, GFS weakly-connected, GFS clopen-connected) set in $(X, E)$ for $i=1,2$, then $f_{u p}\left(F_{\mu}\right)$ is a $G_{F S C}$-connected (respectively, GFS $s$-connected, GFS strongly-connected, GFS weakly-connected, GFS clopen-connected) set in $(Y, K)$ for $i=1,2$. 
Proof. The case of $G F S C_{i}$-connected set $(i=1,2)$ previously proved (see Theorem 4.7 in [11] ). Now, we will prove the case of GFS clopen-connected. Let $F_{\mu}$ be a GFS -clopen connected set in $(X, E)$. Suppose $f_{u p}\left(F_{\mu}\right)$ is not a GFS clopen-connected set in $(Y, K)$. Then, $f_{u p}\left(F_{\mu}\right)$ has non-null proper clopen GFS subset of $J_{\sigma}$. So, there exist $S_{\varepsilon} \in T_{2}$ and $L_{\rho} \in T_{2}^{c}$ such that $J_{\sigma}=f_{u p}\left(F_{\mu}\right) \sqcap S_{\varepsilon}=f_{u p}\left(F_{\mu}\right) \sqcap L_{\rho}$. Since, $f_{u p}$ is injective mapping, then $f_{u p}^{-1}\left(J_{\sigma}\right)=F_{\mu} \sqcap f_{u p}^{-1}\left(S_{\varepsilon}\right)=F_{\mu} \sqcap f_{u p}^{-1}\left(L_{\rho}\right)$. Also, since $S_{\varepsilon} \in T_{2}$ and $L_{\rho} \in T_{2}^{c}$ and $f_{u p}$ is a GFS-continuous mapping, then $f_{u p}^{-1}\left(S_{\varepsilon}\right) \in T_{1}$ and $f_{u p}^{-1}\left(L_{\rho}\right) \in T_{1}^{c}$. Hence, $f_{u p}^{-1}\left(J_{\sigma}\right)$ is non-null proper clopen $G F S$ subset of $F_{\mu}$ which is a contradiction. Therefore, $f_{u p}\left(F_{\mu}\right)$ is a GFS - clopen connected set in $(Y, K)$.

The cases of $G F S C_{3}$-connected and $G_{F S C}$-connected sets we need to the GFS-continuous surjective mapping previously proved (see Theorem 4.8 in [11] ).

Theorem 4.9. Let $\left(X, T_{1}, E\right)$ and $\left(Y, T_{2}, K\right)$ be a GFST-spaces and $f_{u p}:\left(X, T_{1}, E\right) \rightarrow\left(Y, T_{1}, K\right)$ be a $G F S$ injective mapping. If $F_{\mu}$ is a GFS $Q$-connected set in $(X, E)$, then $f_{u p}\left(F_{\mu}\right)$ is a GFS $Q$-connected set in $(Y, K)$.

Proof. Let $F_{\mu}$ be a GFS $Q$-connected set in $(X, E)$. Suppose $f_{u p}\left(F_{\mu}\right)$ is not a GFS $Q$-connected set in $(Y, K)$. Then, there exist two non- null GFS $Q$ separated sets $J_{\sigma}$ and $L_{\rho}$ in $(X, E)$ such that

$$
f_{u p}\left(F_{\mu}\right)=J_{\sigma} \sqcup L_{\rho}, \operatorname{cl}\left(J_{\sigma}\right) \sqcap L_{\rho}=J_{\sigma} \sqcap \operatorname{cl}\left(L_{\rho}\right)=\tilde{0}_{\theta_{Y}} .
$$

Since, $f_{u p}$ is injective mapping, then $f_{u p}^{-1}\left(f_{u p}\left(F_{\mu}\right)\right)=f_{u p}^{-1}\left(J_{\sigma}\right) \sqcup f_{u p}^{-1}\left(L_{\rho}\right)$,

$$
\begin{aligned}
& \left.c l\left(f_{u p}^{-1}\left(J_{\sigma}\right)\right) \sqcap f_{u p}^{-1}\left(L_{\rho}\right) \sqsubseteq f_{u p}^{-1}\left(c l\left(J_{\sigma}\right)\right) \sqcap f_{u p}^{-1}\left(L_{\rho}\right)=f_{u p}^{-1}\left(\operatorname{cl}\left(J_{\sigma}\right) \sqcap L_{\rho}\right)\right)=f_{u p}^{-1}\left(\tilde{0}_{\theta_{Y}}\right)=\tilde{0}_{\theta_{X^{\prime}}} \\
& f_{u p}^{-1}\left(J_{\sigma}\right) \sqcap c l\left(f_{u p}^{-1}\left(L_{\rho}\right)\right) \sqsubseteq f_{u p}^{-1}\left(J_{\sigma} \sqcap f_{u p}^{-1}\left(c l\left(L_{\rho}\right)\right)=f_{u p}^{-1}\left(L_{\rho} \sqcap c l\left(L_{\rho}\right)\right)=f_{u p}^{-1}\left(\tilde{0}_{\theta_{Y}}\right)=\tilde{0}_{\theta_{X}} .\right.
\end{aligned}
$$

This mains that, $f_{u p}^{-1}\left(J_{\sigma}\right), f_{u p}^{-1}\left(L_{\rho}\right)$ are GFS $Q$ separated sets of $F_{\mu}$ in $(X, E)$, which is contradicts of the GFS $Q$-connectedness of $F_{\mu}$ in $(X, E)$. Therefore, $f_{u p}\left(F_{\mu}\right)$ is a GFS $Q$-connected set in $(Y, K)$.

Theorem 4.9. Let $\left(X, T_{1}, E\right)$ and $\left(Y, T_{2}, K\right)$ be a GFST-spaces and $f_{u p}:\left(X, T_{1}, E\right) \rightarrow\left(Y, T_{1}, K\right)$ be a $G F S$ - bijective open mapping. If $G_{\delta}$ is a $G F S C_{i}$-connected(respectively, GFS s-connected, GFS strongly-connected, GFS $Q$-connected, GFS weakly-connected, GFS clopen-connected) set in $(Y, E)$ for $i=$ $1,2,3,4$, then $f_{u p}^{-1}\left(G_{\delta}\right)$ is a $G F S C_{i}$-connected (respectively, GFS s-connected, GFS strongly-connected, GFS $Q$-connected, GFS weakly-connected, GFS-clopen connected) set in $(Y, E)$ for $i=$ $1,2,3,4$.

Proof. The case of GFSC $_{i}$-connected set ( $\left.i=1,2,3,4\right)$ previously proved (see Theorem 4.13 in [11] ). Now, we will prove the case of GFS $s$-connected. Let $G_{\delta}$ is a GFS $s$-connected set in $(Y, K)$. Suppose $f_{u p}^{-1}\left(G_{\delta}\right)$ is not a GFS $s$-connected set in $(X, E)$. Then, there exist two non- null GFS separated sets $H_{v}$ and $K_{\gamma}$ in $(X, E)$ such that $f_{u p}^{-1}\left(G_{\delta}\right)=H_{v} \sqcup K_{\gamma}$. Therefore, there exist two non- null GFS open sets $M_{\psi}$ and $N_{\eta}$ in $(X, E)$ such that $H_{v} \sqsubseteq M_{\psi}$ and $K_{\gamma} \subseteq N_{\eta}$ and $H_{v} \sqcap N_{\eta}=K_{\gamma} \sqcap M_{\psi}=\tilde{0}_{\theta}$. Since, $f_{u p}$ is a GFS surjective mapping, then $f_{u p}\left(f_{u p}^{-1}\left(G_{\delta}\right)\right)=G_{\delta}$ and so $G_{\delta}=f_{u p}\left(H_{v} \sqcup K_{\gamma}\right)=f_{u p}\left(H_{v}\right) \sqcup f_{u p}\left(K_{\gamma}\right)$. Since, $f_{u p}$ is a GFS open mapping, then $f_{u p}\left(M_{\psi}\right)$ and $f_{u p}\left(N_{\eta}\right)$ are non- null GFS open sets in $(Y, K)$ such that $f_{u p}\left(H_{v}\right) \subseteq f_{u p}\left(M_{\psi}\right), f_{u p}\left(K_{\gamma}\right) \subseteq f_{u p}\left(N_{\eta}\right)$. Since, $f_{u p}$ is a GFS injective mapping, then $f_{u p}\left(H_{v}\right) \sqcap f_{u p}\left(N_{\eta}\right)=f_{u p}\left(H_{v} \sqcap N_{\eta}\right)=\tilde{0}_{\theta_{Y}}$ and $f_{u p}\left(K_{\gamma}\right) \sqcap f_{u p}\left(M_{\psi}\right)=$ $\tilde{0}_{\theta_{Y}}$. It follows that $G_{\delta}$ is not a GFS $s$-connected set, a contradiction.

Theorem 4.10. If $F_{\mu}$ and $G_{\delta}$ are intersecting $G F S C_{1}$-(respectively, GFSC $C_{2}$-connected, GFS $s$-connected, GFS weakly-connected, GFS $Q$-connected, GFS strongly-connected) sets in $(X, E)$. Then, $F_{\mu} \sqcup G_{\delta}$ is a

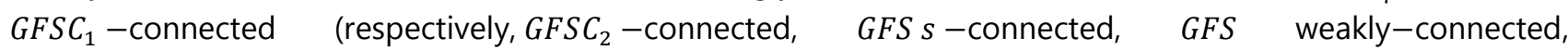
GFS $Q$-connected, GFS strongly-connected) set in $(X, E)$. 
Proof. The cases of $G F S C_{1}$-connected and $G_{F S C}$-connected sets is previously proved (see Theorem 4.9 in [11] ). Now, we will prove the case of GFS $Q$-connected sets. Let $F_{\mu}$ and $G_{\delta}$ are intersecting $G F S Q$-connected sets in $(X, E)$. Suppose $F_{\mu} \sqcup G_{\delta}$ is not a GFS $Q$-connected set. Then, there exist two non- null GFS $Q$-separated sets $H_{v}$ and $K_{\gamma}$ in $(X, E)$ such that $F_{\mu} \sqcup G_{\delta}=H_{v} \sqcup K_{\gamma}$. Therefore, $F_{\mu} \sqcap H_{v}, F_{\mu} \sqcap K_{\gamma}, G_{\delta} \sqcap H_{v}$ and $G_{\delta} \sqcap K_{\gamma}$ are non- null GFS $Q$-separated sets in $(X, E)$ as subsets of $H_{v}$ and $K_{\gamma}$. Since, $F_{\mu}=\left(F_{\mu} \sqcap H_{v}\right) \sqcup$ $\left(F_{\mu} \sqcap K_{\gamma}\right)$ and $G_{\delta}=\left(F_{\mu} \sqcap H_{\nu}\right) \sqcup\left(F_{\mu} \sqcap K_{\gamma}\right)$, then $F_{\mu}$ and $G_{\delta}$ are not $G F S Q$-connected which is a contradiction.

Theorem 4.11. Let $\left\{\left(F_{\mu}\right)_{i}: i \in J\right\}$ be a family of a $G F S C_{1}$-connected (respectively, $G F S C_{2}$-connected, GFS $s$-connected, GFS weakly-connected, GFS $Q$-connected, GFS strongly-connected) sets in $(X, E)$ such that for $i, j \in J$, the GFSSS $\left(F_{\mu}\right)_{i}$ and $\left(F_{\mu}\right)_{j}$ are intersecting. Then, $F_{\mu}=\bigsqcup_{i \in J}\left(F_{\mu}\right)_{i}$ is a $G F S C_{1}$-connected (respectively, GFSC 2 -connected, GFS $S$-connected, GFS weakly-connected, GFS $Q$-connected, GFS strongly-connected) set in $(X, E)$.

Proof. The case of $G_{F S C}$-connected set previously proved (see Theorem 4.11 in [11]). Now, we will prove the

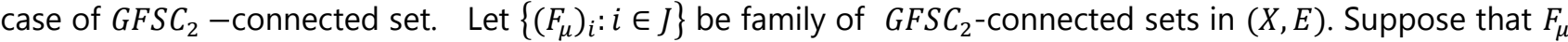
is not a $G F S C_{2}$-connected set in $(X, E)$. Then, there exist two GFS open sets $H_{v}$ and $K_{\gamma}$ in $(X, E)$ such that $F_{\mu}$ ㄷ $H_{v} \sqcup K_{\gamma}, F_{\mu} \sqcap H_{v} \sqcap K_{\gamma}=\tilde{0}_{\theta}, F_{\mu} \sqcap H_{v} \neq \tilde{0}_{\theta}$ and $F_{\mu} \sqcap K_{\gamma} \neq \tilde{0}_{\theta}$.

Now, let $\left(F_{\mu}\right)_{i_{0}}$ be any GFSS of the given family. Then, $\left(F_{\mu}\right)_{i_{0}} \subseteq H_{v} \sqcup K_{\gamma}, H_{v} \sqcap K_{\gamma} \sqsubseteq\left(F_{\mu}\right)_{i_{0}}^{c}$. But, $\left(F_{\mu}\right)_{i_{0}}$ is a GFSC $C_{2}$ connected set. Hence, $\left(F_{\mu}\right)_{i_{0}} \sqcap H_{v}=\tilde{0}_{\theta}$ or $\left(F_{\mu}\right)_{i_{0}} \sqcap K_{\gamma}=\tilde{0}_{\theta}$. Now if $\left(F_{\mu}\right)_{i_{0}} \sqcap H_{v}=\tilde{0}_{\theta}$, we can prove that $\left(F_{\mu}\right)_{i} \sqcap$ $H_{v}=\tilde{0}_{\theta}$ for each $i \in J-\left\{i_{0}\right\}$ and so $F_{\mu} \sqcap H_{v}=\tilde{0}_{\theta}$. This complete the proof.

Corollary 4.1. If $\left\{\left(F_{\mu}\right)_{i}: i \in J\right\}$ is a family of a $G F S C_{1}$-connected (respectively, $G F S C_{2}$-connected, GFS $s$-connected, GFS weakly-connected, GFS $Q$-connected, GFS strongly-connected) sets in $X$ and $\Pi_{i \in J}\left(F_{\mu}\right)_{i} \neq \tilde{0}_{\theta}$, then $F_{\mu}=\bigsqcup_{i \in J}\left(F_{\mu}\right)_{i}$ is a GFSC $C_{1}$-connected (respectively, GFSC $C_{2}$-connected, GFS $s$-connected, GFS weakly-connected, GFS $Q$-connected, GFS strongly-connected) set in $(X, E)$.

The following examples show that Theorem 4.10 fails for $\mathrm{GFSC}_{3}$-connected (respectively, $\mathrm{GFSC}_{4}$-connected) spaces.

Example 4.11. Let $X=\left\{x_{1}, x_{2}\right\}, E=\left\{e_{1}\right\}$ and

$T=\left\{\tilde{0}_{\theta}, \tilde{1}_{\Delta},\left\{\left(e_{1}=\left\{\frac{x_{1}}{4 / 5}, \frac{x_{2}}{2 / 5}\right\}, 4 / 5\right)\right\},\left\{\left(e_{1}=\left\{\frac{x_{1}}{2 / 5}, \frac{x_{2}}{4 / 5}\right\}, 2 / 5\right)\right\},\left\{\left(e_{1}=\left\{\frac{x_{1}}{2 / 5}, \frac{x_{2}}{2 / 5}\right\}, 2 / 5\right)\right\},\left\{\left(e_{1}=\left\{\frac{x_{1}}{4 / 5}, \frac{x_{2}}{4 / 5}\right\}, 4 / 5\right)\right\}\right\}$ be a GFS topology over $(X, E)$. Let $F_{\mu}=\left\{\left(e_{1}=\left\{\frac{x_{1}}{1 / 5}, \frac{x_{2}}{2 / 5}\right\}, 1 / 5\right)\right\}$ and $G_{\delta}=\left\{\left(e_{1}=\left\{\frac{x_{1}}{2 / 5}, \frac{x_{2}}{1 / 5}\right\}, 2 / 5\right)\right\}$. Hence, $F_{\mu} \sqcap G_{\delta} \neq \tilde{0}_{\theta}$ and $F_{\mu}$ and $G_{\delta}$ are $G F S C_{3}$-connected sets in $(X, E)$, but $F_{\mu} \sqcup G_{\delta}$ is not $G F S C_{3}$-connected set in $(X, E)$.

Example 4.12. Let $X=\left\{x_{1}, x_{2}\right\}, E=\left\{e_{1}, e_{2}\right\}$ and

$T=\left\{\tilde{0}_{\theta}, \tilde{1}_{\Delta},\left\{\left(e_{1}=\left\{\frac{x_{1}}{3 / 5}, \frac{x_{2}}{2 / 5}\right\}, 2 / 5\right)\right\},\left\{\left(e_{2}=\left\{\frac{x_{1}}{2 / 5}, \frac{x_{2}}{3 / 5}\right\}, 3 / 5\right)\right\},\left\{\left(e_{1}=\left\{\frac{x_{1}}{3 / 5}, \frac{x_{2}}{2 / 5}\right\}, 2 / 5\right),\left(e_{2}=\left\{\frac{x_{1}}{2 / 5}, \frac{x_{2}}{3 / 5}\right\}, 3 / 5\right)\right\}\right\}$ be a GFS topology over $(X, E)$. Let $F_{\mu}=\left\{\left(e_{1}=\left\{\frac{x_{1}}{3 / 5}\right\}, 2 / 5\right),\left(e_{2}=\left\{\frac{x_{1}}{2 / 5}\right\}, 2 / 5\right)\right\}$ and $G_{\delta}=\left\{\left(e_{1}=\left\{\frac{x_{1}}{1 / 5}, \frac{x_{2}}{2 / 5}\right\}, 1 / 5\right),\left(e_{2}=\right.\right.$ $\left.\left.\left\{\frac{x_{2}}{3 / 5}\right\}, 2 / 5\right)\right\}$. Hence, $F_{\mu} \sqcap G_{\delta} \neq \tilde{0}_{\theta}$ and $F_{\mu}$ and $G_{\delta}$ are $G F S C_{4}$-connected sets in $(X, E)$, but $F_{\mu} \sqcup G_{\delta}$ is not $\mathrm{GFSC}_{4}$-connected set in $(X, E)$.

Theorem 4.12. If $F_{\mu}$ and $G_{\delta}$ are GFS quasi-coincident $G F S C_{3}$-connected (respectively, $G F S C_{4}$-connected) sets in $(X, E)$, then $F_{\mu} \sqcup G_{\delta}$ is a $G F S C_{3}$-connected (respectively, $G F S C_{4}$-connected) set in $(X, E)$.

Proof. As a sample, we will prove the case $G_{F S C}$-connected. Let $F_{\mu}$ and $G_{\delta}$ be $G F S$ quasi-coincident $G_{F S C_{3}}$-connected sets in $(X, E)$. Suppose there exist two non-null GFS open sets $H_{v}$ and $K_{\gamma}$ in $(X, E)$ such that 
$F_{\mu} \sqcup G_{\delta} \sqsubseteq H_{v} \sqcup K_{\gamma}$ and $H_{v} \sqcap K_{\gamma} \sqsubseteq\left(F_{\mu} \sqcup G_{\delta}\right)^{c}$. $\left.\left.G_{\delta}\right)^{c}\right]$
(1) [ we prove that $H_{v} \sqsubseteq\left(F_{\mu} \sqcup G_{\delta}\right)^{c}$ or $K_{\gamma} \sqsubseteq\left(F_{\mu} \sqcup\right.$

Therefore, $F_{\mu} \sqsubseteq H_{v} \sqcup K_{\gamma}, \quad H_{v} \sqcap K_{\gamma} \sqsubseteq F_{\mu}^{c}, \quad G_{\delta} \sqsubseteq H_{v} \sqcup K_{\gamma} \quad$ and $\quad H_{v} \sqcap K_{\gamma} \sqsubseteq G_{\delta}^{c}$. Since, $F_{\mu} \quad$ and $\quad G_{\delta} \quad$ are $G F S C_{3}$-connected, then $\left(H_{v} \sqsubseteq F_{\mu}^{c}\right.$ or $\left.K_{\gamma} \sqsubseteq F_{\mu}^{c}\right)$ and $\left(H_{v} \sqsubseteq G_{\delta}^{c}\right.$ or $\left.K_{\gamma} \sqsubseteq G_{\delta}^{c}\right)$.

Moreover, since $F_{\mu}$ and $G_{\delta}$ are GFS quasi-coincident, there exist $x \in X, e \in E$ such that

$F(e)(x)>1-G(e)(x)$ and $\mu(e)>1-\delta(e)$

Now, consider the following cases:

case 1 . Suppose $H_{v} \sqsubseteq F_{\mu}^{c}$. Then, by (2) we have, $1-H(e)(x) \geq F(e)(x)>1-G(e)(x)$ and $1-v(e) \geq \mu(e)>$ $1-\delta(e) \Rightarrow H(e)(x)<G(e)(x)$ and $v(e)<\delta(e)$.

We claim that, $K_{\gamma} \nsubseteq G_{\delta}^{c}$. For if not, then

$K(e)(x) \leq 1-G(e)(x)<F(e)(x)$ and $\gamma(e) \leq 1-\delta(e)<\mu(e)$

Now by (3) and (4), we have $H(e)(x) \vee K(e)(x)<F(e)(x) \vee G(e)(x)$ and $v(e) \vee \gamma(e)<\mu(e) \vee \delta(e)$ which implies $F_{\mu} \sqcup G_{\delta} \nsubseteq H_{v} \sqcup K_{\gamma}$, this contradicts (1). Hence, $H_{v} \sqsubseteq G_{\delta}^{c}$. Therefore, $H_{v} \sqsubseteq F_{\mu}^{c} \sqcap G_{\delta}^{c}=\left(F_{\mu} \sqcup G_{\delta}\right)^{c}$.

case 2. Suppose $K_{\gamma} \sqsubseteq F_{\mu}^{c}$. Here, we can show as in Case 1 that $H_{v} \nsubseteq G_{\delta}^{c}$. Therefore, $K_{\gamma} \sqsubseteq G_{\delta}^{c}$. Hence, $K_{\gamma} \sqsubseteq G_{\delta}^{c}$. Therefore, $K_{\gamma} \sqsubseteq F_{\mu}^{c} \sqcap G_{\delta}^{c}=\left(F_{\mu} \sqcup G_{\delta}\right)^{c}$. This complete the proof.

Theorem 4.13. Let $\left\{\left(F_{\mu}\right)_{i}: i \in J\right\}$ be a family of GFSC $_{3}$-connected (respectively, $G F S C_{4}$-connected,) sets in $(X, E)$ such that for $i, j \in J$, the GFSSs $\left(F_{\mu}\right)_{i}$ and $\left(F_{\mu}\right)_{j}$ are GFS quasi-coincident. Then, $F_{\mu}=\sqcup_{i \in J}\left(F_{\mu}\right)_{i}$ is a $\mathrm{GFSC}_{3}$-connected (respectively, $\mathrm{GFSC}_{4}$-connected) set in $(X, E)$.

Proof. Let $\left\{\left(F_{\mu}\right)_{i}: i \in J\right\}$ be family of $G F S C_{3}$-connected sets in $(X, E)$. Suppose there exist two GFS open sets $H_{v}$ and $K_{\gamma}$ in $(X, E)$ such that $F_{\mu} \sqsubseteq H_{v} \sqcup K_{\gamma}$ and $H_{v} \sqcap K_{\gamma} \sqsubseteq F_{\mu}^{c}$. Let $\left(F_{\mu}\right)_{i_{0}}$ be any $G F S S$ of the given family. Then, $\left(F_{\mu}\right)_{i_{0}} \sqsubseteq H_{v} \sqcup K_{\gamma}, H_{v} \sqcap K_{\gamma} \sqsubseteq\left(F_{\mu}\right)_{i_{0}}^{c}$. Since, $\left(F_{\mu}\right)_{i_{0}}$ is a $G F S C_{3}$-connected set, we have $H_{v} \sqsubseteq\left(F_{\mu}\right)_{i_{0}}^{c}$ or $K_{\gamma} \sqsubseteq\left(F_{\mu}\right)_{i_{0}}^{c}$. Now, the result follows in view of the facts that $\left(F_{\mu}\right)_{i_{0}} \sqsubseteq H_{v}^{c}$, then $\left(F_{\mu}\right)_{i} \sqsubseteq H_{v}^{c}$ for each $i \in J-\left\{i_{0}\right\}$, since $\left(F_{\mu}\right)_{i_{0}}$ and $\left(F_{\mu}\right)_{i}$ are GFS quasi-coincident $G_{F S C_{3}}$-connected sets, and $H_{v}$ 대 $\left[\Pi_{i \in J}\left(F_{\mu}\right)_{i}\right]^{c}=F_{\mu}^{c}$. Hence, $F_{\mu}$ is a $G F S C_{3}-$ connected. Similarly, if $\left\{\left(F_{\mu}\right)_{i}: i \in J\right\}$ is family of $G F S C_{4}$-connected sets in $(X, E)$ such that for $i, j \in J$, the GFSSS $\left(F_{\mu}\right)_{i}$ and $\left(F_{\mu}\right)_{j}$ are GFS quasi-coincident, then, $F_{\mu}=\bigsqcup_{i \in J}\left(F_{\mu}\right)_{i}$ is a $G F S C_{4}-$ connected set in $(X, E)$. This complete the proof.

Corollary 4.2. Let $\left\{\left(F_{\mu}\right)_{i}: i \in J\right\}$ be a family of a GFSC $C_{3}$-connected (respectively, GFSC $C_{4}$-connected,) sets in $(X, E)$ and $\left(x_{\alpha}, e_{\lambda}\right)$ be a GFS point such that $\alpha>\frac{1}{2^{\prime}} \lambda>\frac{1}{2}$ and $\left(x_{\alpha}, e_{\lambda}\right) \in \Pi_{i \in J}\left(F_{\mu}\right)_{i}$. Then $\sqcup_{i \in J}\left(F_{\mu}\right)_{i}$ is a $\mathrm{GFSC}_{3}$-connected (respectively, $\mathrm{GFSC}_{4}$-connected) set in $(X, E)$.

Proof. Since $\left(x_{\alpha}, e_{\lambda}\right) \in \prod_{i \in J}\left(F_{\mu}\right)_{i}$, then $\left(x_{\alpha}, e_{\lambda}\right) \in\left(F_{\mu}\right)_{i}$ for each $i \in J$. Therefore, $\left(F_{\mu}\right)_{i}$ and $\left(F_{\mu}\right)_{j}$ are GFS quasicoincident for each $i, j \in J$. By Theorem 4.13, $\sqcup_{i \in J}\left(F_{\mu}\right)_{i}$ is a $G F S C_{3}$-connected (respectively, $G F S C_{4}-$ connected ) set in $(X, E)$.

Theorem 4.14. If $F_{\mu}$ is a $G F S C_{3}$-connected (respectively, GFSC 4 -connected, GFS strongly-connected, $G F S Q$-connected) set in $(X, E)$ and $F_{\mu} \sqsubseteq G_{\delta} \sqsubseteq \operatorname{cl}\left(F_{\mu}\right)$, then $G_{\delta}$ is also a $G F S C_{3}$-connected (respectively, $G F S C_{4}$-connected, GFS strongly-connected, GFS $Q$-connected) set in $(X, E)$. In particular $c l\left(F_{\mu}\right)$ is GFSC $_{3}$-connected (respectively, GFSC 4 -connected, GFS strongly-connected, GFS $Q$-connected) set in $(X, E)$. 
Proof. As a sample, we will prove the case $G_{F S C}$-connected. Let $H_{v}$ and $K_{\gamma}$ be $G F S$ open sets in $(X, E)$ such that $G_{\delta} \sqsubseteq H_{v} \sqcup K_{\gamma}$ and $H_{v} \sqcap K_{\gamma} \sqsubseteq G_{\delta}^{c}$. Then, $F_{\mu} \sqsubseteq H_{v} \sqcup K_{\gamma}$ and $H_{v} \sqcap K_{\gamma} \sqsubseteq F_{\mu}^{c}$. Since $F_{\mu}$ is a $G F S C_{3}$-connected set, we have $F_{\mu} \subseteq H_{\nu}^{c}$ or $F_{\mu} \subseteq K_{\gamma}^{c}$. But, if $F_{\mu} \subseteq H_{\nu}^{c}$, then $\operatorname{cl}\left(F_{\mu}\right) \subseteq H_{v}^{c}$ and on the other hand, if $F_{\mu} \subseteq K_{\gamma}^{c}$, then $\operatorname{cl}\left(F_{\mu}\right) \subseteq K_{\gamma}^{c}$. Therefore, $G_{\delta} \subseteq \operatorname{cl}\left(F_{\mu}\right) \subseteq H_{v}^{c}$ or $G_{\delta} \subseteq c l\left(F_{\mu}\right) \subseteq K_{\gamma}^{c}$. Hence, $G_{\delta}$ is a $G F S C_{3}$-connected set in $(X, E)$.

However, the above theorem fails in case of $G F S C_{1}$-connectedness (respectively, $G F S C_{2}$-connectedness, GFS clopen-connectedness, GFS weakly-connectedness, GFS $S$-connectedness) which is a departure from general topology. The following example will illustrate that the closure of a $G F S C_{1}$-connected (respectively, $G F S C_{2}$-connected, GFS clopen-connected, GFS weakly-connected, GFS $s$-connected) set need not be a $G_{F S C_{1}}$-connected (respectively, GFSC 2 -connected, GFS clopen-connected, GFS weakly-connected, GFS $s$-connected).

Example 4.13. Let $X=\left\{x_{1}, x_{2}\right\}, E=\left\{e_{1}, e_{2}\right\}$ and

$T=\left\{\left\{\tilde{0}_{\theta}, \tilde{1}_{\Delta},\left\{\left(e_{1}=\left\{\frac{x_{1}}{1}, \frac{x_{2}}{1}\right\}, 1\right)\right\},\left\{\left(e_{2}=\left\{\frac{x_{1}}{2 / 3}, \frac{x_{2}}{2 / 3}\right\}, 2 / 3\right)\right\},\left\{\left(e_{1}=\left\{\frac{x_{1}}{1}, \frac{x_{2}}{1}\right\}, 1\right),\left(e_{2}=\left\{\frac{x_{1}}{2 / 3}, \frac{x_{2}}{2 / 3}\right\}, 2 / 3\right)\right\}\right\} \quad\right.$ be $\quad$ a $\quad$ GFS topology over $(X, E)$.

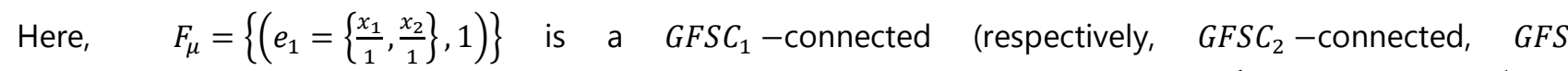
clopen-connected, GFS weakly-connected, GFS $s$-connected) set, but $\operatorname{cl}\left(F_{\mu}\right)=\left\{\left(e_{1}=\left\{\frac{x_{1}}{1}, \frac{x_{2}}{1}\right\}, 1\right),\left(e_{2}=\right.\right.$

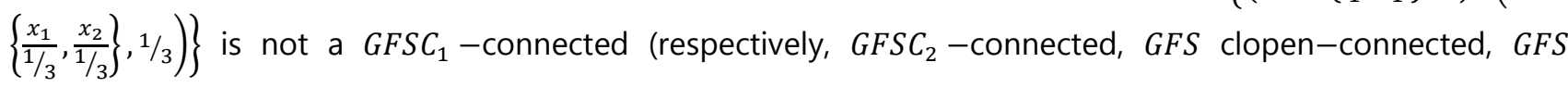
weakly-connected, GFS $s$-connected).

\section{REFERENCES}

[1] N. Ajmal, J. K.. Kohli, Connectedness in fuzzy topological spaces, Fuzzy Sets and Systems. 31(1989) 369-388.

[2] N. Cagman, S. Karatas, S, Enginoglu, Soft Topology comput. Math. Appl. 62 (2011) 351-358.

[3] R. P. Chakraborty and P. Mukherjee, On generalized fuzzy soft topological spaces, Afr. J. Math. Comput. Sci. Res, 8 (2015) 1-11.

[4] C. L. Change, Fuzzy topological spaces, J. Math. Anal. Appl. 24 (1968) 182-190.

[5] 7. U.V. Fatteh and D.S. Bassan, Fuzzy connectedness and its stronger forms, J. Math. Anal. Appl. Ill (1985) 449-464.

[6] A . Kandil, O.A . El-Tantawy, S.A . El-shiekh, Sawsan. S. S. El-Sayed, Fuzzy soft connected sets in fuzzy soft topological spaces I, J. Adv. Math. 8 (12) (2016) 6473-6488 .

[7] S. Karataş, B. Kihç and M. Telliolu, On fuzzy soft connected topological spaces, Journal of Linear and Topological Algebra. 3(4) (2015) 229-240.

[8] A. Kharal. and B. Ahmad, Mappings on fuzzy soft classes, Hindawi Publishing Corporation, Adv. Fuzzy Syst. (2009).

[9] F. H. Khedr, S. A. Abd El-Baki and M. S. Malfi, Results on generalized fuzzy soft topological spaces, African Journal of Mathematics and Computer Science Research. 11(3)(2018) 35-45.

[10] F. H. Khedr, S. A. Abd El-Baki and M. S. Malfi, Generalized fuzzy soft continuity, Appl. Math. Inf. Sci. Submitted. 
[11] F. H. Khedr, S. A. M. AZAB. Abd-Allah and M. S. Malfi, Connectedness on generalized fuzzy soft topological spaces, Journal of New Results in Science. Submitted.

[12] J. Mahanta and P.K. Das, Fuzzy soft topological spaces, Journal of Intelligent \& Fuzzy Systems. 32 (2017) 443-450.

[13] P. K. Maji, R. Biswas and A. R. Roy, Fuzzy soft sets, J. Fuzzy Math. 9 (2001) 589-602.

[14] P. Majumdar and S. K. Samanta, Generalised fuzzy soft sets, Comput. Math. Appl. 59 (2010) 1425-1432.

[15] P.P. Ming and L.Y. Ming, Fuzzy topology I, Neighbourhood structure of a fuzzy point and Moore-Smith convergence, J. Math. Anal. Appl. 76 (1980) 571-599.

[16] D. Molodtsov, Soft set theory-First results, comput. Math. Appl. 37 (1999) 19-31.

[17] P. Mukherjee, Some operators on generalised fuzzy soft topological spaces, Journal of New Results in Science. 9(2015), 57-65.

[18] S. Roy and T. K. Samanta, A note on fuzzy soft topological spaces, Ann. Fuzzy Math. Inform. 3(2) (2011) 305-311.

[19] S. Saha, Local connectedness in fuzzy setting, Simon Stevin. 61(1987) 3-13.

[20] M. Shabir, M. Naz, On soft topological spaces, comput. Math. Appl. 61(2011) 1786-1799.

[21] B. Tanay and M. Burc Kandemir, Topological structure of fuzzy soft sets, comput. Math. Appl. 61(2011) 2952-2957.

[22] L. A. Zadeh, Fuzzy sets, Inform and control. 8 (1965) 338-353.

[23] Zheng Chong You, On connectedness of fuzzy topological spaces, Fuzzy Mathematics 3 (1982) 59-66. 